\title{
A escala de avaliação de demência (DRS) no diagnóstico de comprometimento cognitivo leve e doença de Alzheimer
}

Tese apresentada à Faculdade de Medicina da Universidade de São Paulo para obtenção do título de Doutor em Ciências.

Área de Concentração: Neurologia

Orientador: Prof. Dr. Ricardo Nitrini

SÃO PAULO

2006 
Aos meus pais Roberto e Alba.

À Marina, Letícia e Bárbara. 


\section{AGRADECIMENTOS}

Agradecimento especial ao Prof. Dr. Ricardo Nitrini pela sua valiosa orientação, pela paciência e suas palavras de incentivo, e pela amizade construída com respeito e carinho ao longo desta trajetória.

Agradeço aos colegas do Grupo de Neurologia Cognitiva e do Comportamento pelo companheirismo.

Agradeço a amizade e carinho, em especial, da Dra. Valéria Santoro Bahia.

Agradeço a todos que de alguma maneira contribuíram para a realização deste trabalho, dentre elas, a voluntária Mary Mack Mazzoni pelo carinho e Erly Vieira Soares Junior pela atenção durante estes anos todos.

Agradeço aos pacientes e voluntários que participaram desta tese e me proporcionaram enorme aprendizagem.

Agradeço a Dra.Beatriz Lefèvre, minha mestra, pelo carinho e incentivo.

Agradecimento especial a minha amiga Ana Paula Guanaes Simões Formigoni que, mesmo diante das intercorrências da vida, esteve sempre presente durante esta jornada. 


\section{APRESENTAÇÃO}

A Escala de Avaliação de Demência (Dementia Rating Scale - DRS) tem sido utilizada de maneira sistemática pelo Grupo de Neurologia Cognitiva e do Comportamento (GNCC), do Departamento de Neurologia da Faculdade de Medicina de São Paulo (FMUSP) na avaliação de pacientes com demência há mais de 15 anos.

Em 1990, a partir de um estudo de pacientes com doença de Alzheimer, o GNCC iniciou uma pesquisa na busca de um teste que respondesse às necessidades do trabalho. Na época, Sandra Weintraub e M-Marsel Mesulam estiveram no Brasil e ficamos cientes da sugestão de ambos de que a DRS era um teste promissor para o diagnóstico de demência (Principles of Behavioral Neurology, 1985, p.113). Gentilmente, a Dra. Weintraub encaminhou-nos a DRS e, sob a orientação da Dra. Beatriz Lefèvre, na época, chefe do Serviço de Psicologia da Clínica de Neurologia do Hospital das Clínicas da FMUSP, iniciamos a adaptação para o português, com o cuidado de adaptar os itens desta escala à realidade brasileira. Algumas poucas modificações foram realizadas. Na subescala Iniciativa/Perseveração, no item A3, o sujeito deve repetir PA, KA, LA. Na versão original os monossílabos são Bee, Key e Gee que na tradução literal seriam: abelha, chave e puxa. No item A4, da mesma subescala, o sujeito deve repetir BE, BA, BO; no original, Bee, BA, BO que têm idênticas consoantes e vogais.

No ambulatório do GNCC, na avaliação dos pacientes com hipótese diagnóstica de demência além da DRS são aplicados testes mais específicos que avaliam memória episódica, funções executivas, habilidades construtivas, habilidades vísuo-perceptuais e linguagem. A DRS é um dos primeiros testes, dentro do protocolo, ao qual o paciente é submetido. Observamos que esta opção traz aos pacientes com alta escolaridade uma certa tranqüilidade, amenizando a 
ansiedade da situação de ser avaliado em suas dificuldades, já que os sujeitos costumam achar os itens da DRS muito fáceis. Aos pacientes com baixa escolaridade, a DRS traz a possibilidade de apresentarem respostas corretas, pois vários itens referem-se a situações próximas da vida diária (por exemplo, nomear itens de supermercado).

Os itens mais difíceis, de cada subescala da DRS, são apresentados em primeiro lugar, e quando respondidos corretamente permitem ao examinador que credite como corretas as questões subseqüentes. Este procedimento além de preservar pacientes com ausência de dificuldade na função avaliada, possibilita aos mais comprometidos, que não responderam as primeiras questões, também apresentarem respostas corretas. Desta forma, a DRS avalia as dificuldades dos pacientes juntamente com suas potencialidades.

O fato de a DRS conter subescalas possibilita o acesso, mesmo que parcial, das áreas comprometidas e preservadas que pode auxiliar na complementação da avaliação neuropsicológica, alertando para áreas que devem ser avaliadas mais profundamente. A presença das subescalas também permite a análise da progressão ou não da doença.

Através destes anos pudemos constatar a importância da DRS como um instrumento que permite o acesso a um número maior de informações a respeito do paciente e sua doença, em diferentes graus de intensidade, além de auxiliar no diagnóstico diferencial.

Nos últimos anos, tem sido cada vez maior o número de casos com transtornos cognitivos muito leves que procuram atendimento especializado. As pesquisas sobre o mild cognitive impairment (comprometimento cognitivo leve - CCL) tem aumentado consideravelmente e, entre essas, a busca de instrumentos adequados para o diagnóstico clínico, pois a diferenciação entre 
pacientes com CCL e doença de Alzheimer leve ou entre CCL e idosos normais é tarefa usualmente difícil.

Na dissertação de Mestrado "A escala de avaliação de demência (DRS) no diagnóstico de pacientes com demência de Alzheimer", realizada em 2002 pudemos verificar que a DRS apresenta boa acurácia no diagnóstico de doença de Alzheimer leve. O presente estudo foi motivado pela necessidade de comprovar a acurácia da DRS em nova amostra de pacientes e controles e para investigar este instrumento na avaliação diagnóstica de pacientes com CCL. 


\section{SUMÁRIO}

\section{RESUMO}

Summary

1. INTRODUÇÃO

2. OBJETIVOS 11

2.1. OBJETIVO GERAL 11

2.2. OBJETIVOS ESPECÍFICOS 11

3. MÉTODOS 12

3.1. CASUÍSTICA 12

3.1.1. GRUPO DE PACIENTES 12

3.1.2. GRUPO DE INDIVÍDUOS CONTROLES 13

3.1.3. CASUÍSTICA E CARACTERÍSTICAS DEMOGRÁFICAS 13

3.2. PROCEDIMENTOS 17

3.2.1. AVALIAÇÃO DOS PACIENTES 17

3.2.2. AVALIAÇÃO NEUROPSICOLÓGICA 17

3.2.3. AVALIAÇÃO FUNCIONAL 21

3.3.4. DIAGNÓSTICO 22

3.2.5. AVALIAÇÃO DO GRUPO CONTROLE 23

3.2.6. ESCALA DE AVALIAÇÃO DE DEMÊNCIA (DRS) 23

3.2.7. ANÁLISE ESTATÍSTICA 33

4. RESULTADOS 34

4.1. PACIENTES COM DOENÇA DE ALZHEIMER E CONTROLES 34

4.2. PACIENTES COM DOENÇA DE ALZHEIMER

E COMPROMETIMENTO COGNITIVO LEVE 37

4.3. PACIENTES COM COMPROMETIMENTO COGNITIVO LEVE

E CONTROLES 40

4.4. CORRELAÇÃO ENTRE AS SUBESCALA DA DRS E ALGUNS TESTES NEUROPSICOLÓGICOS 45 
5.1. PACIENTES COM DOENÇA DE ALZHEIMER VERSUS CONTROLES

5.2.PACIENTES COM DOENÇA DE ALZHEIMER VERSUS COMPROMETIMENTO COGNITIVO LEVE

5.3. PACIENTES COM COMPROMETIMENTO

COGNITIVO LEVE VERSUS CONTROLES

5.4.COMPROMETIMENTO COGNITIVO LEVE COMO

UM ESTADO INTERMEDIÁRIO ENTRE IDOSOS

NORMAIS E PACIENTES COM DOENÇA DE ALZHEIMER LEVE 53

5.5. CORRELAÇÃO ENTRE AS SUBESCALAS DA DRS

E ALGUNS TESTES NEUROPSICOLÓGICOS

6. CONCLUSÕES

7. REFERÊNCIAS BIBLIOGRÁFICAS

APÊNDICE 


\section{RESUMO}

Porto CS. A escala de avaliação de demência (DRS) no diagnóstico de comprometimento cognitivo leve e doença de Alzheimer (tese). São Paulo: Faculdade de Medicina, Universidade de São Paulo; 2006.

A Escala de Avaliação de Demência (Dementia Rating Scale -DRS), proposta por Steven Mattis (1988), tem sido bastante utilizada na avaliação de pacientes com demência tanto na atividade clínica como na pesquisa. Consiste de 5 subescalas: Atenção, Iniciativa/Perseveração, Construção, Conceituação e Memória. Neste estudo, a Escala de Avaliação de Demência foi aplicada em 56 pacientes com doença de Alzheimer com demência de intensidade leve; 55 pacientes com diagnóstico de comprometimento cognitivo leve; e, 60 indivíduos controles. $\mathrm{Na}$ diferenciação entre pacientes com doença de Alzheimer e controles a nota de corte de $<128$ demonstrou 90,0\% de sensibilidade e $89,3 \%$ de especificidade; e, entre pacientes com doença de Alzheimer e comprometimento cognitivo leve, a nota de corte foi $<123$ com sensibilidade de $78,2 \%$ e $76,8 \%$ de especificidade. Na diferenciação entre pacientes com comprometimento cognitivo leve e controles, a nota de corte foi de $<134$ com 73,3\% de sensibilidade e 72,7\% de especificidade. A DRS demonstrou ser um instrumento com boa acurácia diagnóstica na discriminação entre pacientes com doença de Alzheimer de intensidade leve e indivíduos controles. A DRS também foi capaz de diferenciar entre pacientes com comprometimento cognitivo leve de controles, e pacientes com comprometiemnto cognitivo leve de pacientes com doença de Alzheimer de intensidade leve. As subescalas Memória e Iniciativa/Perseveração demonstraram maior acurácia diagnóstica em todas as situações analisadas quando comparadas às demais subescalas.

Descritores: delirium,demências,transtorno amnéstico e outros transtornos; doença de Alzheimer; escalas; testes neuropsicológicos; sensibilidade e especificidade. 


\section{SUMMARY}

Porto CS. The dementia rating scale (DRS) in the diagnosis of mild cognitive impairment and Alzheimer's disease (thesis). São Paulo: "Faculdade de Medicina, Universidade de São Paulo"; 2006.

The Dementia Rating Scale (DRS), proposed by Steven Mattis (1988), it has been very used to assess patients with dementia both in clinical practice and research. Consists of 5 subscales: Attention, Initiation/Perseveration, Construction, Conceptualization and Memory. The Dementia Rating Scale was applied to 56 patients with Alzheimer's disease, witha dementia of mild intensity; 55 patients with diagnosis of mild cognitive impairment; and, 60 controls. Between patients with Alzheimer's disease and controls the cutoff score of $<128$ showed a $90.0 \%$ of sensitivity and $89.3 \%$ of specificity; and, between patients with Alzheimer's disease and mild cognitive impairment, the cutoff score was $<123$ with sensitivity of $78.2 \%$ e $76.8 \%$ of specificity. In the analysis between patients with mild cognitive impairment and controls, the cutoff score was $<134$ with $73.3 \%$ of sensitivity and $72.7 \%$ of specificity. The Dementia Rating Scale showed to be a instrument with good diagnostic accuracy in the discrimination between patients with mild Alzheimer's disease and controls. The Dementia Rating Scale also was able to discriminated between patients mild cognitive impairment and controls, and between patients with mild cognitive impairment and mild Alzheimer's disease. The Memory and Initiation/Perseveration subscales showed good diagnostic accuracy in all analysed situations.

Descriptors: delirium, dementia, amnestic, cognitive disorders; Alzheimer's disease; scales; neuropsychologic tests; sensitivity and specificity.

\section{INTRODUÇÃO}

A prevalência de demência dobra a cada 5 anos após os 65 anos, passando de $1 \%$ entre indivíduos sexagenários a 40\% entre idosos com mais de 85 anos (Jorm,1990). A doença de 
Alzheimer (DA) é a forma mais comum de demência no Ocidente, inclusive no Brasil, onde estudo epidemiológico populacional constatou prevalência de demência de 7,1\%, em indivíduos com 65 anos ou mais, tendo sido de $4,9 \%$ a prevalência de DA (Herrera et al., 2002).

A DA caracteriza-se, principalmente nos estágios iniciais, por comprometimento de memória recente enquanto que a memória remota permanece relativamente intacta. Embora a dificuldade de memória seja o principal déficit que compromete as atividades da vida diária, a avaliação de funções cognitivas revela comprometimento de outras funções como atenção, nomeação, raciocínio e habilidades vísuo-espaciais (Mesulam, 2000).

O diagnóstico de demência, particularmente nas fases iniciais do declínio cognitivo, apresenta grande dificuldade, podendo confundir-se com o processo normal de envelhecimento.

Exames complementares auxiliam no diagnóstico nosológico de demência mas é a avaliação neuropsicológica a responsável pelo diagnóstico diferencial entre processos demenciais e outras condições neurológicas ou psiquiátricas.

Os testes utilizados para avaliação de demência devem conter provas consideradas sensíveis para a detecção dos distúrbios cognitivos mais freqüentes na maioria das demências como: memória tanto recente como remota, funções executivas, linguagem, orientação, atenção e praxias (Weintraub, 2000).

A preocupação com o diagnóstico precoce dos quadros demenciais somou-se à necessidade em adaptar testes neuropsicológicos à realidade brasileira e tem levado vários pesquisadores do Brasil a validar teste breves e/ou escalas para a nossa população. Desta 
maneira, o Mini-Exame do Estado Mental (MEEM) (Folstein et al., 1975, Bertolucci et al., 1994, , Brucki et al., 2003), o Consortium to Establish a Registry for Alzheimer's Disease (CERAD) (Morris et al.,1989, Welsh e Mohs,1997, Bertolucci et al, 1998), o Exame de Distúrbios Mentais do Idoso de Cambridge (CAMDEX) (Roth et al., 1986, Bottino et al., 2001), a subescala cognitiva da escala de Avaliação da Doença de Alzheimer (ADAS-cog) (Rosen et al.,1984, Schultz, 1999) e a Bateria de Avaliação Neuropsicológica Breve (NEUROPSI) (Ostrosky-Solis, Ardila e Rosselli, 1999, Abrisqueta-Gomez, 1999) já possuem notas de corte adequadas à população brasileira.

A Escala de Avaliação de Demência (Dementia Rating Scale - DRS) (Mattis,1976, Mattis,1988, Porto et al.,2003) é uma medida do status cognitivo geral, considerado por vários pesquisadores como um instrumento de grande valor para avaliação de pacientes com demência. Desenvolvida, inicialmente, para avaliar habilidades cognitivas de pacientes com doença de Alzheimer (DA), a DRS tem sido freqüentemente utilizada tanto na prática clínica como na pesquisa. Sua aplicação é fácil e relativamente rápida podendo ser administrado, no paciente com demência, em 30 a 40 minutos. As tarefas, no total de 36, são agrupadas em 5 subescalas, as quais avaliam diferentes domínios cognitivos. São elas: Atenção, Iniciativa/Perseveração (I/P), Construção, Conceituação e Memória.

Quando comparado a outras baterias breves, a DRS apresenta algumas vantagens: fornece informações mais detalhadas a respeito das funções cognitivas preservadas e comprometidas do paciente, já que avalia com maior profundidade um maior número de domínios cognitivos (Monsch et al.,1995, Fama et al.,1997); permite a avaliação seqüencial dos pacientes e o grau de declínio (Knox et al., 2003, Miller e Plinskin, 2006), além de apresentar maior capacidade de avaliação das formas graves de doença de Alzheimer (Salmon et al., 1990). 
$\mathrm{O}$ valor do DRS vem sendo reafirmado à medida que mais estudos estão mencionando o uso desta escala no diagnóstico e na discriminação entre pacientes com doença de Alzheimer e pacientes com outras demências como as da doença de Parkinson (Paolo et al., 1995), da Coréia de Huntington (Paulsen et al., 1995) e demência vascular (Kertesz et al.,1994, Lukatela et al.,2000).

Como exemplos, Paolo et al. (1995) demonstraram que os pacientes com doença de Parkinson apresentaram pior desempenho nas provas da subescala Construção quando comparados aos pacientes com DA, enquanto os dados de Paulsen et al. (1995) demonstraram maior prejuízo dos sujeitos com doença de Huntington na subescala I/P em relação aos pacientes com DA. Nos dois estudos, os pacientes com DA apresentaram escores inferiores na subescala Memória. Também as subescalas I/P e Construção foram as mais comprometidas na comparação entre pacientes com demência vascular e sujeitos com DA (Kertesz et al.,1994, Lukatela et al.,2000, Paul et al., 2001).

A importância da DRS e particularmente de suas subescalas em estabelecer correlações clínico-topográficas tem sido descrita em trabalhos que relacionam esta escala a exames de imagem como Ressonância Magnética e SPECT (Karbe et al., 1994, Stout et al., 1996, Fama et al., 1997).

Apesar de muito utilizada, as propriedades psicométricas da DRS, em sua maioria, não foram bem documentadas (Monsch et al., 1995, Hoffer et al., 1996, Lucas et al., 1998). 
A DRS foi desenvolvida a partir de um estudo prospectivo realizado por Coblentz et al. (1973), já que, segundo os autores, poucos testes poderiam diferenciar níveis de habilidades dentro do grupo de pacientes com demência, e fornecer uma medida para o estudo longitudinal de declínio cognitivo. Os autores aplicaram a DRS em 11 indivíduos normais e 20 pacientes com síndrome mental orgânica. Correlacionou-se a DRS à Escala Wechsler de Inteligência para Adultos (WAIS) (Wechsler, 1993) e ao subteste Pares Associados da Escala Wechsler de Memória (Wechsler, 1945). Os sujeitos controles, com quociente de inteligência total no WAIS acima de 85 e escores nos Pares Associados dentro da média, apresentaram escores de 140 a 144 no total da DRS.

Montgomery e Costa (1982) (apud Spreen e Strauss,1998) aplicaram a DRS em uma amostra de 85 idosos normais, com idade variando de 65 a 81 anos, com média de idade igual a 74 e média de escolaridade de 12,4. Neste estudo, os autores constataram uma média de escore total de 137,3 e desvio padrão de 6,9.

Em outro estudo realizado por Montgomery e Costa (1983) (apud Mattis, 1988), no qual foram examinados grupos de pacientes com depressão, desordens psicológicas, comprometimento cerebral focal e demência, a acurácia diagnóstica do ponto de corte da DRS foi investigada. Nenhum dos pacientes com depressão, $12 \%$ dos pacientes com transtornos psicológicos e $36 \%$ pacientes com comprometimento cerebral focal apresentaram escore menor que 123. Por outro lado, $62 \%$ dos pacientes com demência apresentaram escore abaixo deste valor.

Os valores citados nestes trabalhos são limitados. A amostra utilizada por Coblentz é considerada muito pequena e tanto o nível socio-econômico como a escolaridade, dos sujeitos da 
amostra de Montgomery, são muito altos (Spreen e Strauss, 1998). Portanto seus dados não são considerados representativos, o que tem levado pesquisadores a validar esta escala em populações específicas.

Schmidt et al. (1994) aplicaram a DRS em 1.001 voluntários austríacos, com idade de 50 a 80 anos. Os critérios de inclusão foram ausência de doenças graves ou neuropsiquiátricas, queixa de esquecimentos, evidência de dependência de álcool ou drogas e anormalidades laboratoriais importantes. As notas de corte foram apresentadas em percentis e, segundo os autores, as normas propostas neste trabalho provaram apresentar alta sensibilidade desde que ajustadas aos níveis de escolaridade e idade.

Em estudo realizado por Vangel e Lichtenberg (1995) (apud Spreen e Strauss, 1998) do qual participaram 90 indivíduos, a maioria afro-americanos, sem queixa de alterações cognitivas, a nota de corte proposta de 125 , foi capaz de classificar corretamente $87 \%$ da amostra, separando os indivíduos com prejuízo cognitivo e sem prejuízo cognitivo.

Monsch et al. (1995) realizaram um estudo com o objetivo de avaliar a validade clínica da DRS em detectar pacientes com DA. Fizeram parte deste estudo 254 pacientes com DA e 105 sujeitos idosos normais. A nota de corte encontrada foi 129, com sensibilidade de $98 \%$ e especificidade de $97 \%$. Fatores demográficos como idade e escolaridade influenciaram os escores da DRS. Concluiu-se, assim, que a DRS é um teste psicométrico clinicamente válido para detectar pacientes com DA.

Vanotti et al. (2001) aplicaram a DRS em 385 idosos normais, voluntários, pertencentes a população rioplatense (Argentina), com idade de 50 a 91 anos e escolaridade de 0 a 19 anos. O 
escore total médio da DRS foi 135,2 ( \pm 5,39). Tanto a idade como a escolaridade interferiram nos escores da DRS.

Estudos recentes têm demonstrado a influência da idade e escolaridade no desempenho da DRS sugerindo que uma única nota de corte não é apropriada para todos os grupos de idosos (Bennett et al., 1997, Lucas et al.,1998, Bank et al., 2000, Chan et al. , 2003, Rilling et al., 2005).

No Brasil, a DRS vem sendo utilizada, pelo Grupo de Neurologia Cognitiva e do Comportamento da Clínica de Neurologia do Hospital das Clínicas da Faculdade de Medicina da Universidade de São Paulo, desde 1990, de maneira sistemática, na avaliação de pacientes com demência. Através destes anos pudemos constatar a importância da DRS como um instrumento que permite o acesso a um número maior de informações a respeito do paciente e sua doença, em diferentes graus de intensidade, além de permitir estudos seqüenciais de pacientes e auxiliar no diagnóstico diferencial.

Porto et al. (2003) realizaram estudo na população brasileira. A DRS foi aplicada em 41 pacientes com doença de Alzheimer provável, de intensidade leve (média de idade $=71,59 \pm$ 8,41 ; média de escolaridade $=9,07 \pm 5,31$ ) e 60 controles (média de idade $=69,65 \pm 8,49$; média de escolaridade $=8,05 \pm 4,62$ ). O escore $<123$ demonstrou sensibilidade de 91,7\% e especificidade de $87,8 \%$, apresentando desta forma, boa acurácia diagnóstica. Para a análise das variáveis idade e escolaridade, os pacientes com DA e controles, foram divididos em três grupos de idade (50 a 65 anos, 66 a 75 anos e maior que 75 anos) e três níveis de escolaridade ( 1 a 4 anos, 5 a 11 anos e maior que 11 anos de escolaridade). Idade e escolaridade interferiram no escore total e nos escores das subescalas da DRS, tendo a variável escolaridade apresentado interferência mais acentuada. 
A influência da baixa escolaridade e do analfabetismo, na DRS, foi analisada por Foss et al. (2005) em estudo com 62 idosos normais, com idade variando de 64 a 77 anos, e divididos em cinco grupos, segundo a escolaridade: 15 e 16 anos, 11 e 12 anos, 8 e 9 anos, 4 anos e analfabetos. $\mathrm{O}$ grupo de analfabetos obteve resultados inferiores ao dos grupos escolarizados no escore total da DRS e em todas as suas subescalas. Não houve correlação significativa quanto à idade em relação aos resultados da escala.

Alguns trabalhos têm utilizado a DRS na avaliação de pacientes com declínio cognitivo que inclua o comprometimento cognitivo leve (CCL). O conceito de CCL (mild cognitive impairment), proposto por Petersen et al. $(1999,2001)$, é descrito como um estado cognitivo entre normalidade e demência e o risco de conversão para DA é $12 \%$ ao ano (Petersen et al., 2001). Pode ser classificado de acordo com três subtipos: amnéstico, múltiplo domínios e comprometimento de uma única função cognitiva diferente de memória. Os critérios para CCL amnéstico são: queixa de memória, escores abaixo da média em testes de memória, status cognitivo global preservado, ausência de dificuldades em atividades da vida diária, e ausência de demência. Caso o indivíduo apresente leve dificuldade em outras funções cognitivas, na presença ou não de déficit de memória, é classificado como CCL de múltiplos domínios; e receberá a classificação de CCL de outra função, se o comprometimento for em outra função cognitiva que não memória (Winblad et al., 2004).

Salmon et al. (2002), em estudo em que, embora a designação mild cognitive impairment (MCI) não tenha sido utilizada, foram avaliados pacientes com o que os autores denominaram DA com comprometimento cognitivo muito leve ("very mildly impaired individuals"). A amostra composta de 110 sujeitos, com escore no MEEM igual ou maior a 24 e diagnóstico clínico de 
DA, foi avaliada anualmente em um período de pelo menos 3 anos. O diagnóstico de DA foi confirmado em 98 dos 110 (89\%) pacientes com comprometimento muito leve. Os autores concluíram que a utilização da DRS associada a medidas neuropsicológicas de memória episódica e fluência verbal demonstrou excelente sensibilidade (= 96\%) e especificidade (=93\%) quando comparados com controle idosos.

Com o objetivo de investigar a heterogeneidade cognitiva de uma amostra de idosos, com diagnóstico de DA provável leve, presente ou ausente, Charchat-Fichman (2003) utilizou além do MEEM (Brucki et al., 2003) e do teste de aprendizagem verbal auditiva de Rey (Diniz et al., 2000), como critério para diagnóstico clínico. Os pacientes foram diagnosticados como com Comprometimento de Memória Associado à Idade (AAMI), Declínio Cognitivo Associado ao Envelhecimento (AACD), Comprometimento Cognitivo Leve do tipo amnéstico, demência questionável e DA provável.

Boeve et al. (2003), realizaram estudo em uma amostra de 111 indivíduos, com idade de 90 a 100 anos a qual foi submetida a avaliação neurológica, neuropsicológica e funcional. Os sujeitos foram diagnosticados como normais (56 indivíduos), ou com CCL (13 pacientes) ou com dementes (42 pacientes). Pacientes com demência, quando comparados a indivíduos normais apresentaram pior desempenho nos escores do MEEM, da DRS e do Teste das Trilhas - Parte A. Resultado semelhante foi encontrado na comparação entre pacientes com demência e CCL. Entretanto, os escores nestes testes não foram diferentes na comparação entre controles e CCL. Os escores no Free and Cued Selective Reminding Test (FCSRT) e na subescala Memória da DRS diferiram entre os três grupos. 
Geda et al. (2004) aplicaram o Inventário Neuropsiquiátrico (NPI), a DRS e o MEEM com o objetivo de determinar o perfil neuropsiquiátrico de pacientes com CCL, comparando com sujeitos controles e pacientes com DA leve. Os escores do MEEM e da DRS assim como do NPI foram significativamente diferentes entre os três grupos.

Griffith et al. (2006) estudaram a taxa de conversão de CCL para DA em um período de 2 anos. A subescala I/P da DRS e o sub-teste Reprodução Visual da Escala Wechsler de Memória III (WMS-III) foram aplicados em 49 pacientes com CCL. O escore abaixo de 37, na subescala I/P, e o escore de retenção abaixo de $26 \%$, no teste Reprodução Visual, identificaram corretamente a conversão para DA com 76,9\% de sensibilidade e 91,7 \% de especificidade.

Em outro estudo realizado por Griffith et al. (2006), foram utilizados a DRS, o sub-teste de Memória Lógica do Wechsler Memory Scale (WMS) e a fluência verbal de categorias fonêmicas em 26 idosos com epilepsia, em 26 pacientes com CCL, e em 26 controles. Idosos com epilepsia apresentaram desempenho inferior ao dos controles em todos os testes neuropsicológicos enquanto pacientes com CCL tiveram desempenho inferior na DRS total e na subescala I/P e também no teste de fluência verbal de categorias fonêmicas.

Foi possível verificar que o valor da DRS na discriminação entre pacientes com DA e pacientes com CCL ou entre pacientes com CCL e indivíduos controles não está ainda bem estabelecido. A contribuição deste trabalho é verificar o desempenho de pacientes com CCL, comparado a pacientes com DA e com sujeitos controles, na versão brasileira da DRS assim como em suas subescalas. 


\section{OBJETIVOS}

\subsection{Objetivo Geral}

Avaliar a acurácia da Escala de Avaliação de Demência (Dementia Rating Scale - DRS ) no diagnóstico de pacientes com demência de intensidade leve da doença de Alzheimer e de pacientes com comprometimento cognitivo leve.

\subsection{Objetivos específicos}

Confirmar a acurácia diagnóstica do escore total da DRS assim como de suas subescalas no diagnóstico de pacientes com demência leve da doença de Alzheimer.

Verificar a capacidade da DRS na discriminação entre sujeitos controles e pacientes com comprometimento cognitivo leve (CCL).

Verificar a capacidade da DRS na discriminação entre pacientes com demência de intensidade leve da doença de Alzheimer e pacientes com comprometimento cognitivo leve. 
Verificar a correlação entre as subescalas da DRS e alguns testes que avaliam domínios neuropsicológicos por elas abrangidos.

\section{MÉTODOS}

\subsection{Casuística}

\subsubsection{Grupo de pacientes}

Foram avaliados 111 pacientes ( 70 mulheres e 41 homens), com idade de 54 a 87 anos (média $=72,52 \pm 7,11)$, escolaridade de 3 a 17 anos (média $=10,86 \pm 4,55$ ), com queixa de memória, atendidos pelos membros do Grupo de Neurologia Cognitiva e do Comportamento do Departamento de Neurologia do Hospital das Clínicas da Faculdade de Medicina da Universidade de São Paulo.

Critérios de inclusão:

1) queixa subjetiva de transtorno de memória confirmada por informante;

2) presença de informante capaz de fornecer dados fidedignos;

3) ter se submetido a exame neurológico, exames complementares e avaliação neuropsicológica;

4) ter diagnóstico clínico de doença de Alzheimer (DA) com demência de intensidade leve ou de comprometimento cognitivo leve (CCL).

Critérios de exclusão:

1) demência de intensidade moderada ou grave;

2) demência de outra etiologia; 
3) presença de outras condições com potencial para comprometer cognição como depressão, uso de fármacos psicotrópicos e doenças psiquiátricas.

\subsubsection{Grupo de indivíduos controles}

O grupo controle com 60 sujeitos foi composto de cônjuges ou acompanhantes, não consangüíneos, de pacientes, ou voluntários pertencentes à comunidade, sem queixas subjetivas ou de informante quanto a presença de transtornos de memória e independentes em relação às atividades da vida diária.

Foram excluídos sujeitos com doença neurológica, alcoolismo, depressão, outros transtornos psiquiátricos, distúrbios visuais ou auditivos não corrigidos, comprometimento motor e indivíduos que consumiam drogas psicotrópicas que poderiam comprometer funções cognitivas. Doenças crônicas como hipertensão arterial, diabete melito e cardiopatia, se compensadas, não foram critérios de exclusão.

\subsubsection{Casuística e características demográficas}

Foram avaliados 56 pacientes com DA provável, com demência de intensidade leve, com idade de 54 a 84 anos, com escolaridade de 3 a 17 anos, sendo 35 mulheres e 21 homens; 55 indivíduos com diagnóstico de CCL ( 24 com CCL amnéstico e 31 com CCL de múltiplos domínios), com idade de 54 a 87 anos, escolaridade de 3 a 17 anos, sendo 35 mulheres e 20 homens; e, 60 controles, com idade de 51 a 82, escolaridade de 1 a 16 anos, sendo 42 mulheres e 18 homens.

Não houve diferença estatisticamente significativa entre os três grupos (DA, CCL e controles) quanto ao gênero $(\mathrm{p}=0,652)$. A idade $(\mathrm{p}=0,007)$ e escolaridade $(\mathrm{p}=0,015)$ entre DA, CCL e controles foi estatisticamente diferente entre os grupos. 
Os dados demográficos entre pacientes com DA e controles, entre pacientes com DA e CCL, e entre pacientes com CCL e controles estão nas Tabelas 1,2,3,4,5 e 6.

Tabela 1: Idade e Escolaridade de DA e controles

\begin{tabular}{lccc}
\hline & DA & Controles & $\mathbf{p}$ \\
\hline $\mathbf{N}$ & 56 & 60 & \\
Idade & & & \\
Média & 72,98 & 68,90 & 0,003 \\
DP & 9,62 & 7,48 & \\
Mediana & 73,00 & 69,50 & \\
& & & \\
Escolaridade & & & \\
Média & 9,62 & 10,19 & 0,185 \\
DP & 4,68 & 4,73 & \\
Mediana & 8,50 & 11,00 & \\
N= número de sujeitos da amostra; DA = doença de Alzheimer; \\
DP= desvio padrão; $\mathrm{p}=$ valor de significância $(\mathrm{p}<0,05)$
\end{tabular}

$\mathrm{DP}=$ desvio padrão; $\mathrm{p}=$ valor de significância $(\mathrm{p}<0,05)$

Tabela 2: Gênero de DA e controles

\begin{tabular}{llll}
\hline Sexo & DA & Controles & p \\
\hline N & 56 & 60 & \\
F & 35 & 42 & 0,393 \\
M & 21 & 18 & \\
\\
N= número de sujeitos da amostra; DA = doença de Alzheimer; \\
p=valor de significância $(\mathrm{p}<0,05)$
\end{tabular}

Tabela 3: Idade e Escolaridade de DA e CCL

DA $\quad$ CCL $\quad p$




\begin{tabular}{lccc} 
Idade & & & \\
Média & 72,98 & 72,05 & 0,327 \\
DP & 9,62 & 6,81 & \\
Mediana & 73,00 & 72,00 & \\
& & & \\
Escolaridade & & & \\
Média & 9,62 & 12,13 & 0,004 \\
DP & 4,68 & 4,07 & \\
Mediana & 8,50 & 12,00 & \\
\hline
\end{tabular}

$\mathrm{N}=$ número de sujeitos da amostra; $\mathrm{DA}=$ doença de Alzheimer; $\mathrm{CCL}=$ comprometimento cognitivo leve; $\mathrm{DP}=$ desvio padrão; $\mathrm{p}=$ valor de significância $(\mathrm{p}<0,05)$

Tabela 4: Gênero de DA e controles

\begin{tabular}{llll}
\hline Sexo & DA & CCL & p \\
\hline N & & & \\
F & 56 & 55 & \\
M & 35 & 35 & 0,901 \\
\hline
\end{tabular}

$\mathrm{N}=$ número de sujeitos da amostra; DA = doença de Alzheimer; $\mathrm{CCL}=$ comprometimento cognitivo leve; $\mathrm{p}=$ =valor de significância $(\mathrm{p}<0,05)$

Tabela 5: Idade e Escolaridade de CCL e controles 


\begin{tabular}{lccc}
\hline & $\mathbf{C C L}$ & Controles & $\mathbf{p}$ \\
\hline $\mathbf{N}$ & 55 & 60 & \\
& & & \\
Idade & & & \\
Média & 72,05 & 68,90 & 0,029 \\
DP & 6,81 & 7,48 & \\
Mediana & 72,00 & 69,50 & \\
& & & \\
Escolaridade & & & \\
Média & 12,13 & 10,19 & 0,108 \\
DP & 4,07 & 4,73 & \\
Mediana & 12,00 & 11,00 & \\
\hline
\end{tabular}

$\mathrm{N}=$ número de sujeitos da amostra; $\mathrm{CCL}=$ comprometimento cognitivo leve; $\mathrm{DP}=$ desvio padrão; $\mathrm{p}=$ valor de significância $(\mathrm{p}<0,05)$

Tabela 6: Gênero de CCL e controles

\begin{tabular}{lccc}
\hline Sexo & CCL & Controles & p \\
\hline N & & & \\
F & 55 & 60 & \\
M & 35 & 42 & 0,469 \\
\hline
\end{tabular}

$\mathrm{N}=$ número de sujeitos da amostra; $\mathrm{CCL}=$

comprometimento cognitivo leve; $\mathrm{p}=$ valor de significância $(\mathrm{p}<0,05)$

\subsection{Procedimentos}

\subsubsection{Avaliação dos pacientes}


Todos os pacientes foram submetidos a exames laboratoriais, exames de neuroimagem (Tomografia Computadorizada ou Ressonância Magnética), Mini-Exame do Estado Mental (MEEM) (Folstein et al., 1975; Brucki et al, 2003), Bateria Breve de Rastreio Cognitivo (BBRC) (Nitrini et al., 1994, 2005), avaliação neuropsicológica extensa e avaliação funcional.

\subsubsection{Avaliação neuropsicológica}

A avaliação neuropsicológica, dos pacientes, foi composta dos seguintes testes:

(a) Memória visual e verbal:

- Reprodução Visual: subteste da Escala Wechsler de Memória - Revisada (WMS-R) (Wechsler, 1987). Consiste de 4 cartões com figuras abstratas impressas, sendo que três contêm uma figura cada e o quarto cartão contém duas figuras. Cada figura é mostrada ao sujeito por 10 segundos e, após cada exposição, é solicitado ao indivíduo que desenhe o que recorda das figuras; depois de 30 minutos, solicita-se novamente que desenhe o que recorda das figuras. Avalia tanto a memória visual imediata como a tardia. O escore total do teste é de 41 pontos.

- Evocação da Figura Complexa de Rey (Spreen e Strauss, 1998, Rey, 1998). Avalia memória visual e habilidades construtivas. O teste consiste na cópia de uma figura complexa que deve ser novamente reproduzida sem auxílio do modelo, após 30 minutos. Escore total igual a 36 pontos em cada fase. 
- Memória Lógica: subteste da WMS-R (Wechsler, 1987). Este teste é composto por duas estórias curtas, lidas pelo examinador, e após a leitura de cada uma é solicitada a lembrança imediata. Anotam-se quantos itens são recordados dentre os 25 itens que cada estória contém. Depois de 30 minutos solicita-se a evocação tardia das estórias e novamente se anotam quantos itens são recordados. É usual que a recordação imediata seja denominada Memória Lógica I enquanto a tardia é denominada Memória Lógica II. O escore máximo de cada fase é de 50 pontos.

- Teste de Aprendizagem Verbal Auditiva de Rey (RAVLT) (Spreen e Strauss, 1998,Diniz et al., 2000). O teste consiste na leitura de uma lista de 15 palavras. Após a leitura solicita-se, ao sujeito, a evocação das palavras. Este procedimento é repetido 5 vezes. Anotam-se o número de palavras evocadas em cada tentativa. A pontuação máxima do somatório de palavras evocadas nas 5 tentativas é 75 . Uma nova lista de 15 palavras é lida e após a apresentação desta segunda lista pede-se a evocação da primeira lista. Após intervalo de tempo de 30 minutos é solicitado novamente a evocação da primeira lista.

(b) Habilidades construtivas

- Cubos: subteste da Escala Wechsler de Inteligência para Adultos (WAIS) (Wechsler, 1993). São apresentados ao sujeito cubos vermelhos e brancos. A tarefa consiste em usar os cubos para construir réplicas de modelos de desenhos impressos. O escore máximo da prova é de 36 pontos, transformados através de uma tabela em notas ponderadas.

- Cópia da Figura Complexa de Rey (Spreen e Strauss, 1998, Rey, 1998). Ver acima. 
(c) Percepção Visual

- Teste de Organização Visual de Hooper (Hooper, 1983). O teste é composto por trinta figuras, constituídas pelas partes dos objetos dispostos como em um quebra-cabeça. A tarefa consiste em reconstruir mentalmente a figura. Estas figuras, uma de cada vez, são mostradas ao sujeito que deverá nomear cada uma delas. O total de respostas corretas, 30 no máximo.

- Matrizes Progressivas de Raven (Raven et al., 1988, Lezak, 1995). Consiste no total de 36 itens, agrupados em 3 grupos de 12 figuras cada um. É um teste de múltipla escolha em que o examinando deve inferir qual a regra relacionada e apontar qual é a figura correta entre quatro alternativas. A pontuação máxima é de 36 pontos.

(d) Linguagem

- Teste de Nomeação de Boston (Goodglass e Kaplan, 1987, Radanovic et al., 2004): É um teste de nomeação e consiste na apresentação de 60 desenhos que variam, quanto a freqüência no vocabulário, entre alta (exemplo: árvore) e baixa (exemplo: ábaco). A pontuação máxima possível é 60 pontos.

(e) Funções Executivas

- Teste das Trilhas (Spreen e Strauss, 1998). O teste é composto de duas partes, A e B. Na parte A, o sujeito deve conectar consecutivamente círculos numerados. Em uma outra folha (parte B), é solicitado que o indivíduo conecte seqüencialmente, círculos com 
números e com letras, alternando entre as duas seqüências (exemplo: 1,A, 2,B). O escore, de cada versão, é o total de tempo, em segundos, utilizado pelo sujeito para completar a prova.

- Teste de Stroop (Spreen e Strauss, 1998). O teste é composto por 3 provas. Na primeira prova o sujeito deve nomear retângulos de cores (rosa, verde, azul e marrom); na segunda prova, é solicitada a leitura de palavras pintadas de cores diferentes; e, na terceira prova, é solicitado, ao examinando, falar a cor que a palavra está pintada inibindo a leitura da palavra. A pontuação é o tempo e o número de erros em cada prova.

- Wisconsin Card Sorting Test (WCST) (Spreen e Strauss, 1998). A tarefa compõe-se de 4 cartões estímulo (um triângulo vermelho, duas estrelas verdes, três cruzes amarelas e quatro círculos azuis) e 64 cartões respostas. Ao sujeito é solicitado que combine cada um dos 64 cartões, com os cartões estímulos. O critério para combinar os cartões deve ser escolhido pelo sujeito. Cabe ao examinador dizer apenas se a resposta está correta ou errada. As categorias envolvidas na tarefa são cores, formas e números. Sem o conhecimento do sujeito, o examinador determina a categoria correta. Após 10 respostas corretas, o critério é modificado pelo examinador, novamente, sem o sujeito ser notificado da mudança. O escore depende da capacidade do indivíduo de perceber tanto as categorias que podem ser utilizadas como as regras propostas pelo examinador.

- Fluência verbal para categorias fonêmicas (F.A.S.) (Spreen e Strauss, 1998). O teste consiste na geração, do maior número de palavras, no período de um minuto, com a letra F, excluindo nomes próprios, a mesma palavra com sufixo diferente e números. O mesmo 
procedimento é utilizado com a letra A e a letra S. A pontuação da prova é a soma do número de palavras geradas com cada uma das letras.

\subsubsection{Avaliação Funcional}

Quanto às informações de atividades da vida diária foi aplicado, ao informante, a Escala de Atividades de Vida Diária de Pfeffer (Pfeffer et al., 1982, Nitrini, 2005). O escore total da prova é de 30 pontos.

\subsubsection{Diagnóstico}

O diagnóstico de DA com demência de intensidade leve e o de CCL foi realizado por dois neurologistas que não tiveram acesso aos resultados da Escala de Avaliação de Demência de Mattis (DRS) e da BBRC, e baseou-se na anamnese obtida com o paciente e com informantes, exame físico e neurológico, resultados de exames laboratoriais e de imagem, escores do MEEM e nos resultados dos seguintes testes neuropsicológicos: habilidades construtivas (Cubos (WAIS)), memória (Teste de Aprendizagem Verbal Auditiva de Rey (RAVLT) (somatória das tentativas e evocação das palavras após 30 minutos)), linguagem (Teste de Nomeação de Boston) e funções executivas (fluência verbal para categorias fonêmicas - F.A.S. e o Teste das Trilhas (versão A e B)).

O diagnóstico de demência de intensidade leve foi baseado nos critérios do Manual de Diagnóstico e Estatística de Transtornos Mentais, $3^{\mathrm{a}}$ Edição, revisada (DSM-III-R) (1987) e o 
diagnóstico de DA provável baseou-se nos critérios do National Institute of Neurological Disorders and Communicative Disorders and Stroke-Alzheimer's Disease and Related Disorders Association (NINCDS-ADRDA) (McKhann et al., 1984).

O diagnóstico de CCL baseou-se nos critérios de Petersen et al. (1999, 2001). São eles: queixa subjetiva de memória, preferencialmente confirmada por um informante; desempenho inferior a 1,5 desvio padrão em tarefas de memória, comparado com grupo controle pareado por idade e escolaridade; funcionamento cognitivo global preservado; independência quanto às atividades da vida diária; ausência de demência.

\subsubsection{Avaliação do grupo controle}

Todos os sujeitos incluídos no grupo controle foram submetidos ao MEEM e o Questionário de Queixas Subjetivas de Memória (MAC-Q) (Mattos et al., 2003) ou Informant Questionnaire on Cognitive Decline in the Elderly (IQCODE) (Jorm, 1994), solicitado a um informante. Para o MEEM foram utilizadas as notas de corte propostas no estudo de Brucki et al. (2003), segundo a escolaridade ( 1 a 4 anos de escolaridade $=24,85 \pm 3,03 ; 5$ a 8 anos $=26,57 \pm$ $1,51 ; 9$ a 11 anos $=28,75 \pm 1,26 ; \mathrm{e}=12$ anos de escolaridade $=27,17 \pm 1,94)$. Para o MAC-Q a nota de corte foi 22 (Xavier et al., 2002) e para o IQCODE 3,41 (Bustamante et al., 2003).

\subsubsection{Escala de Avaliação de Demência (Dementia Rating Scale - DRS)}

Em todos os pacientes e controles foi aplicada a versão em português da DRS (Porto et al., 2003), composta de 36 tarefas distribuídas em 5 subescalas: Atenção, Iniciativa/Perseveração, Construção, Conceituação e Memória segundo as orientações do autor. A apresentação das 
provas obedeceu a uma ordem fixa, sendo que, apenas os testes da subescala Atenção não estão agrupados em sequiência, pois servem como tarefas distratoras para a subescala Memória. Os testes mais difíceis são apresentados, dentro de cada subescala, em primeiro e segundo lugar e, quando respondidos corretamente permitem ao examinador que credite as tarefas subseqüentes, desta subescala, como corretas. Este procedimento tem a vantagem de diminuir o tempo total de testagem para indivíduos relativamente intactos.

O número de pontos creditados para respostas corretas varia de acordo com as tarefas e a soma dos pontos, em cada subescala, fornece um escore parcial referente àquela subescala. Os escores parciais são: Atenção, 37 pontos; Iniciativa/Perseveração, 37 pontos; Construção, 06 pontos; Conceituação, 39 pontos; e Memória, 25 pontos. O escore total é de 144 pontos.

Os itens avaliados, inseridos dentro de cada subescala, são:

\section{Atenção}

\section{Números}

O paciente deve repetir uma seqüência de números em ordem direta e inversa. A pontuação é o número de dígitos que o paciente foi capaz de repetir em ordem direta e inversa. O número máximo de pontos, a serem creditados, é somente $4 \mathrm{em}$ ordem direta e $4 \mathrm{em}$ ordem inversa. Pontuação máxima 8.

2. Respostas a duas ordens consecutivas 
O examinador pede ao paciente para seguir dois comandos consecutivos (por exemplo: abrir a boca e fechar os olhos). Se o paciente responder corretamente os itens 3 e 4 serão considerados corretos. Pontuação máxima 2.

\section{Respostas a ordens verbais simples}

O paciente deve realizar comandos simples (exemplo: abra a boca), em um total de 4 . Pontuação máxima 4.

\section{Imitação}

O examinador executa uma ação simples, como fechar os olhos e, o paciente deve imitálo. Pontuação máxima 4.

\section{Contagem de letras A}

Diante de algumas letras (vogais e consoantes) o paciente deverá contar o número de letras "A" que está vendo. Esta é uma tarefa distratora da subescala Memória. Pontuação máxima 6.

\section{Contagem de letras A}

O mesmo procedimento da tarefa anterior. Também é uma prova distratora da subescala Memória. Pontuação máxima 5.

\section{Leitura da Lista}

É solicitado ao paciente que leia uma lista de palavras quatro vezes para que ele se lembre de cada uma delas. Marcar um ponto para cada vez que as 5 palavras forem lidas 
corretamente. Esta prova é uma prova de apresentação de itens que serão solicitados na tarefa de memória de reconhecimento verbal. Pontuação máxima 4.

\section{Combinação de Desenhos}

O paciente deve combinar 4 desenhos apresentados na parte superior da folha com outros 4 que estão na parte inferior. Qualquer instrução é válida desde que ela capacite o paciente a fazer a combinação dos desenhos. Todos os desenhos devem ser combinados. Um ponto para cada desenho combinado corretamente. Estes desenhos serão solicitados novamente na prova de memória de reconhecimento visual. Pontuação máxima 4.

\section{Iniciativa/Perseveração}

\section{Fluência Verbal}

O paciente deve dizer o nome de todas as coisas que se pode comprar em um supermercado no prazo de um minuto. Se 14 itens ou mais forem mencionados o examinador pode omitir as três tarefas subseqüentes, atribuindo ao paciente a contagem máxima. Pontuação máxima 20.

\section{Nomear peças do vestuário}

O examinador pede ao paciente que nomeie todas as peças do vestuário que está vendo, ou seja, tanto as dele como as do examinador. Marcar o número de itens mencionados no prazo de um minuto. Pontuação máxima 8.

\section{Repetição de Sílabas}

O paciente deve repetir, quatro vezes, as sílabas PA,KA,LA. Pontuação máxima 1. 


\section{Repetição de Sílabas}

O mesmo procedimento da prova anterior com as sílabas: BE,BA,BO. Pontuação máxima 1.

\section{Movimentos Duplos Alternados}

O examinador deve demonstrar ao paciente: palma da mão esquerda para cima, palma da mão direita para baixo. Em seguida, troque a posição das mãos simultaneamente 5 vezes. Marcar um ponto no caso do correto posicionamento das mãos durante 5 alternações consecutivas. Pontuação máxima 1.

\section{Movimentos Duplos Alternados}

O examinador demonstra: mão direita fechada, com a palma voltada para baixo, dedos da mão esquerda estendidos com a palma voltada para baixo. Alternar a posição das mãos simultaneamente várias vezes. Marcar um ponto para 5 alterações consecutivas corretas. Pontuação máxima 1.

\section{Movimentos Duplos Alternados}

O examinador deve tamborilar os dedos alternadamente, demonstrando o movimento com o dedo indicador de cada mão. Marcar um ponto para 10 alternações consecutivas. Pontuação máxima 1.

16. Desenho Gráfico-motor (1) 
O paciente deve copiar uma seqüência de um desenho simples. Se o paciente realizar a cópia corretamente atribuir a contagem máxima nas 3 etapas seguintes.Pontuação máxima 1

17. Desenho Gráfico-motor (2)

O paciente deve copiar um círculo. Pontuação máxima 1.

18. Desenho Gráfico-motor (3)

O paciente deve copiar um "X". Pontuação máxima 1.

19. Desenho Gráfico-motor (4)

O paciente deve copiar uma linha alternada de círculos e "X". Pontuação máxima 1.

\section{Construção}

20. Cópia (1)

Copiar uma figura geométrica: um losango dentro de um quadrado. Caso o paciente copie corretamente, receberá a pontuação máxima nas etapas seguintes $(2,3,4,5,6)$. Pontuação máxima 1.

21. Cópia (2)

Copiar uma figura geométrica: quadrado ao lado de um losango. Pontuação máxima 1.

22. Cópia (3)

Copiar um losango. Pontuação máxima 1. 
23. Cópia (4)

Copiar um quadrado. Pontuação máxima 1.

24. Cópia (5)

Copiar quatro linhas verticais. Pontuação máxima 1.

25. Cópia (6)

Escrever seu nome. Pontuação máxima 1.

\section{Conceituação}

26. Semelhanças

O paciente deverá dizer qual a semelhança, por exemplo, entre a banana e a maçã. Será computado 2 pontos para conceito abstrato e um ponto para conceito concreto. Se o paciente obtiver 6 pontos nesta prova, receberá a contagem máxima nos itens subseqüentes. Pontuação máxima 8.

27. Raciocínio Indutivo

O paciente deverá nomear o que as pessoas comem e depois dizer qual a semelhança entre elas. O mesmo para o que as pessoas vestem e o que as pessoas usam para se locomover. Pontuação máxima 3.

28. Diferenças

O examinador nomeia três coisas e o paciente deve dizer qual delas não combina com as demais. Pontuação máxima 3. 


\section{Semelhanças - Múltipla Escolha}

O examinador pergunta qual a semelhança entre, por exemplo, a maçã e a banana, oferecendo ao paciente três alternativas. É creditado um ponto para conceito concreto e dois pontos para abstrato. Pontuação máxima 8 .

30. Igualdades e Diferenças

Diante de três figuras geométricas, o paciente deve dizer quais são as duas figuras semelhantes e qual a figura diferente. Pontuação máxima 16.

31. Elaboração da frase

O paciente deve formar uma frase simples usando as palavras homem e carro. Pontuação máxima 1.

\section{Memória}

32. Lembrança da frase

O paciente deve ler, em voz alta, uma frase e, após algumas provas distratoras, é solicitado a evocá-la. Pontuação máxima 4.

33. Lembrança da frase

O paciente é solicitado a evocar a frase que ele havia elaborado anteriormente. Pontuação máxima 3.

34. Orientação 
O examinador pergunta ao paciente informações como: dia, mês, ano; nome do presidente, do governador e prefeito; nome do hospital, cidade. Pontuação máxima 9.

35. Memória de reconhecimento verbal

É apresentado ao paciente pares de palavras, entre as quais ele deve escolher qual já havia lido anteriormente. A lista de palavras foi apresentada previamente (item 7). Pontuação máxima 5 .

36. Memória de reconhecimento visual

As figuras que foram combinadas pelo paciente, no item 8 , são apresentadas aos pares. $\mathrm{O}$ paciente deve escolher, entre as duas figuras, qual delas ele já havia visto (item 8).

A aplicação da DRS, nos grupos estudados, foi feita de forma individual em uma única sessão. O tempo de aplicação para o grupo de pacientes foi, em média, 40 minutos e para o grupo controle de 20 a 30 minutos.

Todos os indivíduos concordaram em participar do estudo e assinaram o termo de consentimento informado devidamente aprovado pelo Comitê de Ética e Pesquisa do Hospital das Clínicas da Faculdade de Medicina de São Paulo. 


\subsubsection{Análise Estatística}

Para avaliar associações entre as variáveis categóricas e o desfecho foi utilizado o teste do Qui-quadrado de Pearson. Quando as variáveis eram contínuas as comparações foram feitas utilizando, para duas amostras, o Teste de Mann-Whitney, para mais de duas, o teste de KruskallWallis.

Foram realizados cálculos de sensibilidade e especificidade de cada subescala e da escala total. O escore de corte, através de Curvas ROC (receiver operator characteristics), foi definido como aquele em que a sensibilidade e a especificidade apresentaram os valores mais próximos entre si.

Para as correlações entre subescalas e testes neuropsicológicos foi utilizado o teste de correlação de Spearman. Valores de $\mathrm{r}_{\mathrm{s}}$ superiores 0,75 indicaram correlação muito boa; entre 0,5 e 0,74 boa, e abaixo de 0,5 ruim.

Foi considerado risco menor ou igual a 5\% para erros tipo I e risco beta maior ou igual a $20 \%$ para erro tipo II.

Toda a análise estatística foi realizada usando o Programa Statistical Package for the Social Sciences, para Windows, versão 10.0 (SPSS). 


\section{RESULTADOS}

\subsection{Pacientes com DA e controles}

A média do escore total da DRS nos pacientes com DA foi de 113,82 (desvio padrão (DP) de $\pm 12,41)$ e do grupo controle $136,21 \pm 6,31$. Constatou-se diferença significativa quanto ao escore médio da pontuação total entre o grupo de DA e controles $(p=0,001)$ e nas subescalas Atenção $(p=0,001)$, Iniciativa/Perseveração $(\mathrm{I} / \mathrm{P})(\mathrm{p}=0,001)$, Construção $(\mathrm{p}=0,004)$, Conceituação $(\mathrm{p}=0,001)$ e Memória $(\mathrm{p}=0,001)$ (Tabela 7). 
Tabela 7: Desempenho de DA e controles na DRS

\begin{tabular}{|c|c|c|c|}
\hline DRS & DA & Controles & $\mathbf{p}$ \\
\hline $\mathbf{N}$ & 56 & 60 & \\
\hline \multicolumn{4}{|l|}{ Atenção } \\
\hline Média & 34,89 & 35,85 & \multirow{3}{*}{0,001} \\
\hline DP & 1,71 & 1,29 & \\
\hline Mediana & 35,00 & 36,00 & \\
\hline \multicolumn{4}{|l|}{$I / P$} \\
\hline Média & 29,25 & 35,95 & \multirow{3}{*}{$=0,001$} \\
\hline DP & 5,78 & 1,88 & \\
\hline Mediana & 29,00 & 37,00 & \\
\hline \multicolumn{4}{|c|}{ Construção } \\
\hline Média & 5,50 & 5,91 & \multirow{3}{*}{0,004} \\
\hline DP & 1,02 & 0,42 & \\
\hline Mediana & 6,00 & 6,00 & \\
\hline \multicolumn{4}{|c|}{ Conceituação } \\
\hline Média & 29,37 & 34,50 & \multirow{3}{*}{$=0,001$} \\
\hline DP & 6,13 & 4,11 & \\
\hline Mediana & 29,00 & 36,00 & \\
\hline \multicolumn{4}{|l|}{ Memória } \\
\hline Média & 14,83 & 24,00 & \multirow{3}{*}{$=0,001$} \\
\hline DP & 3,86 & 1,16 & \\
\hline Mediana & 14,00 & 24,00 & \\
\hline \multicolumn{4}{|l|}{ Total } \\
\hline Média & 113,82 & 136,21 & \multirow{3}{*}{$=0,001$} \\
\hline DP & 12,41 & 6,31 & \\
\hline Mediana & 115,00 & 138,00 & \\
\hline
\end{tabular}

DRS = Escala de Avaliação de Demência $; \mathrm{DA}=$ doença de Alzheimer; $\mathrm{I} / \mathrm{P}=$ Iniciativa/Perseveração; $\mathrm{DP}=$ desvio padrão da média; $\mathrm{p}=$ valor de significância. Teste de Mann Whitney $(\mathrm{p}<0,05)$ 
Foram definidas notas de corte para cada subescala e para o escore total da DRS utilizando-se as curvas ROC ( receiver operating characteristics) (Figura 1).

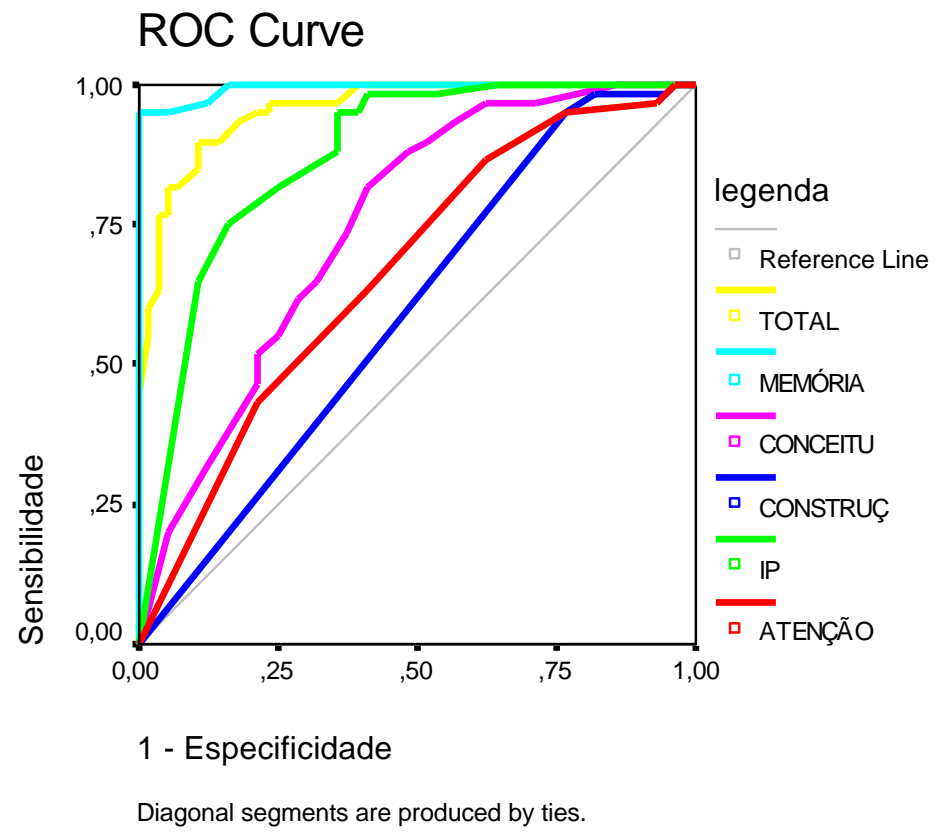

Figura 1: Curva ROC (receiver operating characteristics) do escore total e das subescalas da DRS entre controles e pacientes com DA.

A nota de corte obtida para o total da DRS foi $<128$ com sensibilidade de $90,0 \%$ e especificidade de $89,3 \%$. A acurácia global da DRS assim como de cada subescala foi analisada através das áreas sob as curvas. Observou-se que, além do escore total, as subescalas Memória, 
I/P e Conceituação foram as provas com melhor poder discriminatório. As áreas sob as curvas com seu valor de significância, notas de corte com as respectivas sensibilidade e especificidade estão na Tabela 8 .

Tabela 8: Área sob a curva, valor de significância, notas de corte, sensibilidade e especificidade do escore total e das subescalas da DRS, entre pacientes com DA e controles.

\begin{tabular}{lcccccc}
\hline \multicolumn{1}{c}{ DRS } & ASC (EP) & $\mathrm{p}$ & $\begin{array}{c}\text { Máximo } \\
\text { de pontos }\end{array}$ & $\begin{array}{c}\text { Nota de } \\
\text { Corte* }\end{array}$ & $\begin{array}{c}\text { Sensibilidade } \\
\%\end{array}$ & $\begin{array}{c}\text { Especificidade } \\
\%\end{array}$ \\
\hline Total & $0,957 \pm 0,016$ & $=0,001$ & 144 & $<128$ & 90,0 & 89,3 \\
Atenção & $0,667 \pm 0,050$ & 0,002 & 37 & $<36$ & 63,3 & 58,0 \\
I/P & $0,871 \pm 0,034$ & $=0,001$ & 37 & $<35$ & 81,7 & 75,0 \\
Construção & $0,593 \pm 0,053$ & 0,085 & 6 & $<6$ & 95,0 & 23,2 \\
Conceituação & $0,748 \pm 0,046$ & $=0,001$ & 39 & $<32$ & 73,3 & 62,5 \\
Memória & $0,994 \pm 0,004$ & $=0,001$ & 25 & $<22$ & 95,0 & 94,6 \\
\hline
\end{tabular}

DRS = Escala de Avaliação de Demência; ASC = área sob a curva; EP = erro padrão; $\mathrm{p}$ = valor de significância ; I/P = Iniciativa/Perseveração.

* indivíduos com escore abaixo do escore de corte são considerados com prejuízo

\subsection{Pacientes com DA e CCL}

Comparou-se o desempenho, na DRS, dos pacientes com DA e CCL. O escore total médio dos indivíduos com CCL foi de $128,43 \pm 7,35$. Houve diferença estatisticamente diferente na pontuação total $(p=0,001)$, entre o grupo de DA e de CCL, e nas subescalas Atenção $(p=$ 0,002), I/P ( $\mathrm{p}=0,001)$, Construção $(\mathrm{p}=0,005)$, Conceituação $(\mathrm{p}=0,001)$ e Memória $(\mathrm{p}=$ 0,001) (Tabela 9). 
Tabela 9: Desempenho de DA e CCL na DRS

\begin{tabular}{lccc}
\hline DRS & DA & $\mathbf{C C L}$ & $\mathbf{p}$ \\
\hline $\mathbf{N}$ & $\mathbf{5 6}$ & $\mathbf{5 5}$ & \\
Atenção & & & \\
Média & 34,89 & 35,85 & \\
DP & 1,71 & 1,12 & 0,002 \\
Mediana & 35,00 & 36,00 & \\
I/P & & & \\
Média & 29,25 & 34,14 & \\
DP & 5,78 & 3,42 & $=0,001$ \\
Mediana & 29,00 & 36,00 & \\
Construção & & & \\
Média & 5,5 & 5,94 & \\
DP & 1,02 & 0,22 & 0,005 \\
Mediana & 6,00 & 6,00 & \\
Conceituação & & & \\
Média & 29,37 & 33,41 & \\
DP & 6,13 & 4,95 & $=0,001$ \\
Mediana & 29,00 & 34,00 & \\
Memória & & & \\
Média & 14,83 & 19,07 & \\
DP & 3,86 & 2,93 & $=0,001$ \\
Mediana & 14,00 & 19,00 & \\
Total & & & \\
Média & 113,82 & 128,43 & \\
DP & 12,41 & 7,35 & $=0,001$ \\
Mediana & 115,00 & 128,00 & \\
\hline & & & \\
& &
\end{tabular}

DRS = Escala de Avaliação de Demência; DA = doença de Alzheimer; $\mathrm{CCL}=$ comprometimento cognitivo leve; I/P = Iniciativa/Perseveração; DP = desvio padrão da média; $\mathrm{p}=$ valor de significância. Teste de Mann Whitney $(\mathrm{p}<0,05)$ 
Através das curvas ROC (Figura 2) estabeleceu-se que a nota de corte, para o escore total da DRS, entre DA e CCL, foi < 123 com sensibilidade igual a 78,2\% e especificidade de $76,8 \%$. Tanto o escore total como as subescalas Memória, I/P e Conceituação foram as com maior poder discriminatório, entre DA e CCL. As áreas sobre a curva, valor de significância e notas de corte com as respectivas sensibilidade e especificidade estão na Tabela 10.

\section{Curva ROC}

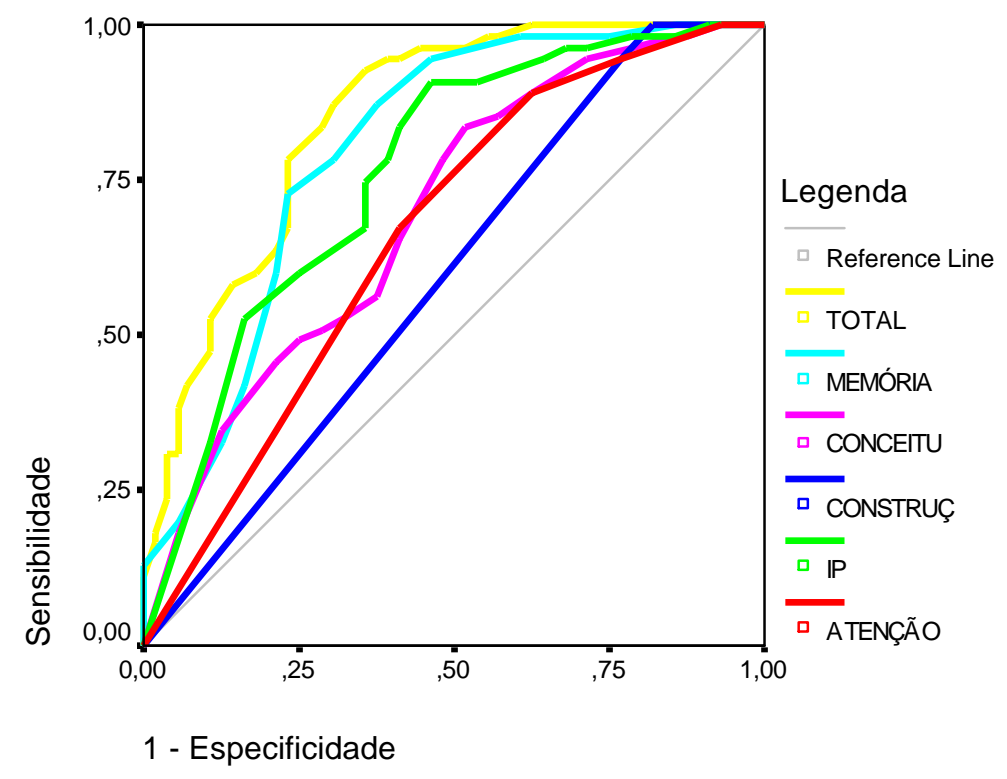

Figura 2: Curva ROC (receiver operating characteristics) do escore total e das subescalas de DRS entre DA e CCL.

Tabela 10: Área sob a curva, valor de significância, notas de corte, sensibilidade e especificidade do escore total e das subescalas da DRS, entre pacientes com DA e CCL. 


\begin{tabular}{lcccccc}
\hline \multicolumn{1}{c}{ DRS } & ASC (EP) & $\mathrm{p}$ & $\begin{array}{c}\text { Máximo } \\
\text { de pontos }\end{array}$ & $\begin{array}{c}\text { Nota de } \\
\text { Corte* }\end{array}$ & $\begin{array}{c}\text { Sensibilidade } \\
\%\end{array}$ & $\begin{array}{c}\text { Especificidade } \\
\%\end{array}$ \\
\hline Total & $0,663 \pm 0,052$ & $=0,001$ & 144 & $<123$ & 78,2 & 76,8 \\
Atenção & $0,762 \pm 0,045$ & 0,003 & 37 & $<36$ & 67,3 & 58,9 \\
I/P & $0,594 \pm 0,054$ & $=0,001$ & 37 & $<33$ & 74,5 & 64,3 \\
Construção & $0,698 \pm 0,049$ & 0,089 & 6 & $<6$ & 94,5 & 23,2 \\
Conceituação & $0,801 \pm 0,042$ & $=0,001$ & 39 & $<31$ & 65,5 & 58,9 \\
Memória & $0,850 \pm 0,036$ & $=0,001$ & 25 & $<17$ & 78,2 & 69,6 \\
\hline
\end{tabular}

DRS = Escala de Avaliação de Demência; $\mathrm{ASC}=$ área sob a curva; $\mathrm{EP}=$ erro padrão; $\mathrm{p}=$ valor de significância ; I/P = Iniciativa/Perseveração.

* indivíduos com escore abaixo do escore de corte são considerados com prejuízo

\subsection{Pacientes com CCL e controles}

Entre pacientes com CCL e indivíduos controles, houve diferença significativa, na pontuação média, do escore total $(\mathrm{p}=0,001)$ e das subescalas I/P $(\mathrm{p}=0,001)$ e Memória $\quad(\mathrm{p}=$ 0,001). Não houve diferença estatisticamente significativa nas subescalas Atenção $(p=0,774)$, Construção $(p=0,931)$ e Conceituação $(p=0,306)$ (Tabela 11). 
Tabela 11: Desempenho de CCL e controles na DRS

\begin{tabular}{lccc}
\hline DRS & CCL & Controles & $\mathbf{p}$ \\
\hline $\mathbf{N}$ & $\mathbf{5 5}$ & $\mathbf{6 0}$ & \\
Atenção & & & \\
Média & 35,85 & 35,85 & \\
DP & 1,12 & 1,29 & 0,774 \\
Mediana & 36,00 & 36,00 & \\
I/P & & & \\
Média & 34,14 & 35,95 & \\
DP & 3,42 & 1,88 & $=0,001$ \\
Mediana & 36,00 & 37,00 & \\
Construção & & & \\
Média & 5,94 & 5,91 & \\
DP & 0,22 & 0,42 & 0,931 \\
Mediana & 6,00 & 6,00 & \\
Conceituação & & & \\
Média & 33,41 & 34,50 & \\
DP & 4,95 & 4,11 & 0,306 \\
Mediana & 34,00 & 36,00 & \\
Memória & & & \\
Média & 19,07 & 24,00 & \\
DP & 2,93 & 1,16 & $=0,001$ \\
Mediana & 19,00 & 24,00 & \\
Total & & & \\
Média & 128,43 & 136,21 & \\
DP & 7,35 & 6,31 & $=0,001$ \\
Mediana & 128,00 & 138,00 & \\
\hline & & &
\end{tabular}


DRS = Escala de Avaliação de Demência; $\mathrm{CCL}=$ comprometimento cognitivo leve; $\mathrm{I} / \mathrm{P}=$ Iniciativa/Perseveração; $\mathrm{DP}=$ desvio padrão da média; $\mathrm{p}=$ valor de significância. Teste de Mann Whitney $(\mathrm{p}<0,05)$

Foram definidas nota de corte para o escore total e as subescalas da DRS através das curvas ROC (Figura 3).

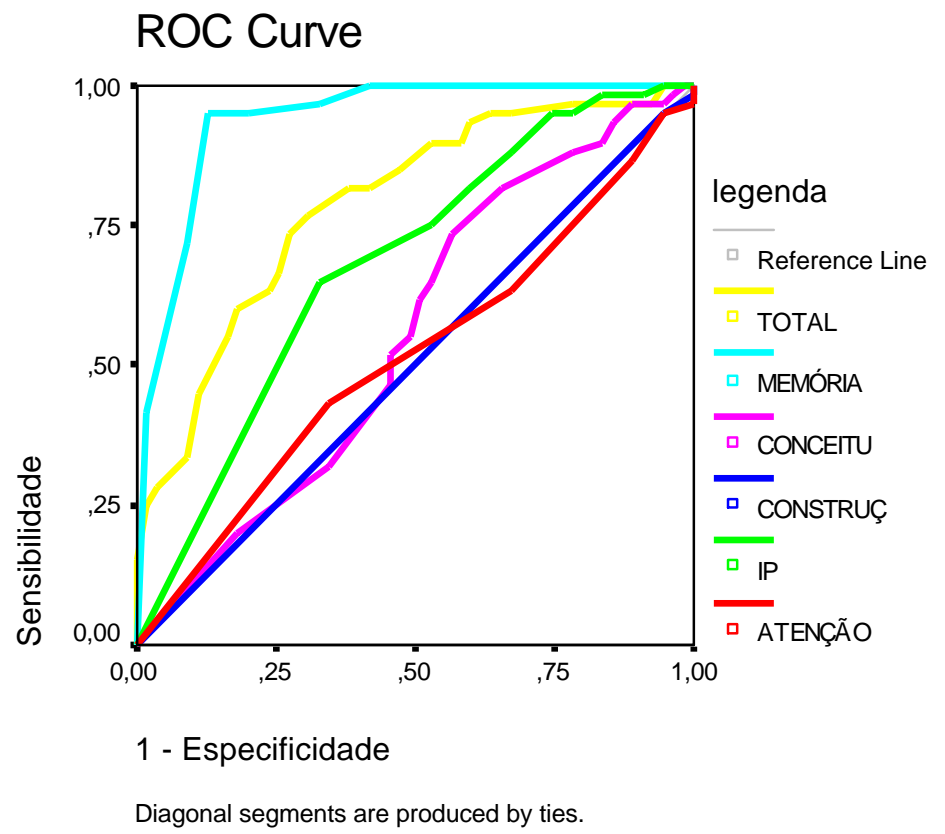

Figura 3: Curva ROC (receiver operating characteristics) do escore total e das subescalas da DRS entre sujeitos controles e CCL.

A nota de corte encontrada foi $<134$ com sensibilidade de $73,3 \%$ e $72,7 \%$ de especificidade. Além do escore total, as subescalas Memória e I/P demonstraram boa acurácia 
diagnóstica. As áreas sob as curvas com seu valor de significância, notas de corte com as respectivas sensibilidade e especificidade estão na Tabela 12.

Tabela 12: Área sob a curva, valor de significância, notas de corte, sensibilidade e especificidade do escore total e das subescalas da DRS, entre pacientes com CCL e controles.

\begin{tabular}{lcccccc}
\hline \multicolumn{1}{c}{ DRS } & ASC (EP) & $\mathrm{p}$ & $\begin{array}{c}\text { Máximo } \\
\text { de pontos }\end{array}$ & $\begin{array}{c}\text { Nota de } \\
\text { Corte* }\end{array}$ & $\begin{array}{c}\text { Sensibilidade } \\
\%\end{array}$ & $\begin{array}{c}\text { Especificidade } \\
\%\end{array}$ \\
\hline Total & $0,788 \pm 0,042$ & $=0,001$ & 144 & $<134$ & 73,3 & 72,7 \\
Atenção & $0,515 \pm 0,054$ & 0,784 & 37 & $<36$ & 63,3 & 32,7 \\
I/P & $0,681 \pm 0,050$ & 0,001 & 37 & $<37$ & 65,0 & 67,3 \\
Construção & $0,502 \pm 0,054$ & 0,973 & 6 & $<6$ & 95,0 & 5,5 \\
Conceituação & $0,555 \pm 0,055$ & 0,310 & 39 & $<35$ & 55,0 & 50,9 \\
Memória & $0,938 \pm 0,023$ & $=0,001$ & 25 & $<23$ & 95,0 & 87,3 \\
\hline
\end{tabular}

DRS = Escala de Avaliação de Demência; ASC $=$ área sob a curva $; \mathrm{EP}=$ erro padrão; $\mathrm{p}=$ valor de significância $; \mathrm{I} / \mathrm{P}$ = Iniciativa/Perseveração.

*indivíduos com escore abaixo do escore de corte são considerados com prejuízo

As notas de corte dos grupos DA e sujeitos controles, DA e CCL e CCL e controles estão na Tabela 13.

Tabela 13: notas de corte dos grupos de DA e controles, DA e CCL e CCL e controles.

DRS Máximo de Pontos DA e Controles * DA e CCL * CCL e Controles *




\begin{tabular}{lcccc} 
Total & 144 & $<128$ & $<123$ & $<134$ \\
Atenção & 37 & $<36$ & $<36$ & $<36$ \\
I/P & 37 & $<35$ & $<34$ & $<37$ \\
Construção & 6 & $<6$ & $<6$ & $<6$ \\
Conceituação & 39 & $<32$ & $<31$ & $<35$ \\
Memória & 25 & $<22$ & $<17$ & $<23$ \\
\hline
\end{tabular}

DRS = Escala de Avaliação de Demência; $\mathrm{I} / \mathrm{P}=$ Iniciativa/Perseveração; DA = doença de Alzheimer; $\mathrm{CCL}=$ comprometimento cognitivo leve.

*indivíduos com escore abaixo do escore de corte são considerados com prejuízo

O desempenho da DRS foi comparado entre pacientes com DA, CCL e controles e constataram-se diferença significativa no escore total $(p=0,001)$ e nas subescalas Atenção $(p=$ 0,001), Iniciativa/Perseveração $(p=0,001)$, Construção $(p=0,001)$, Conceituação $(p=0,001)$ e Memória $(\mathrm{p}=0,001)$ (Figura 4).

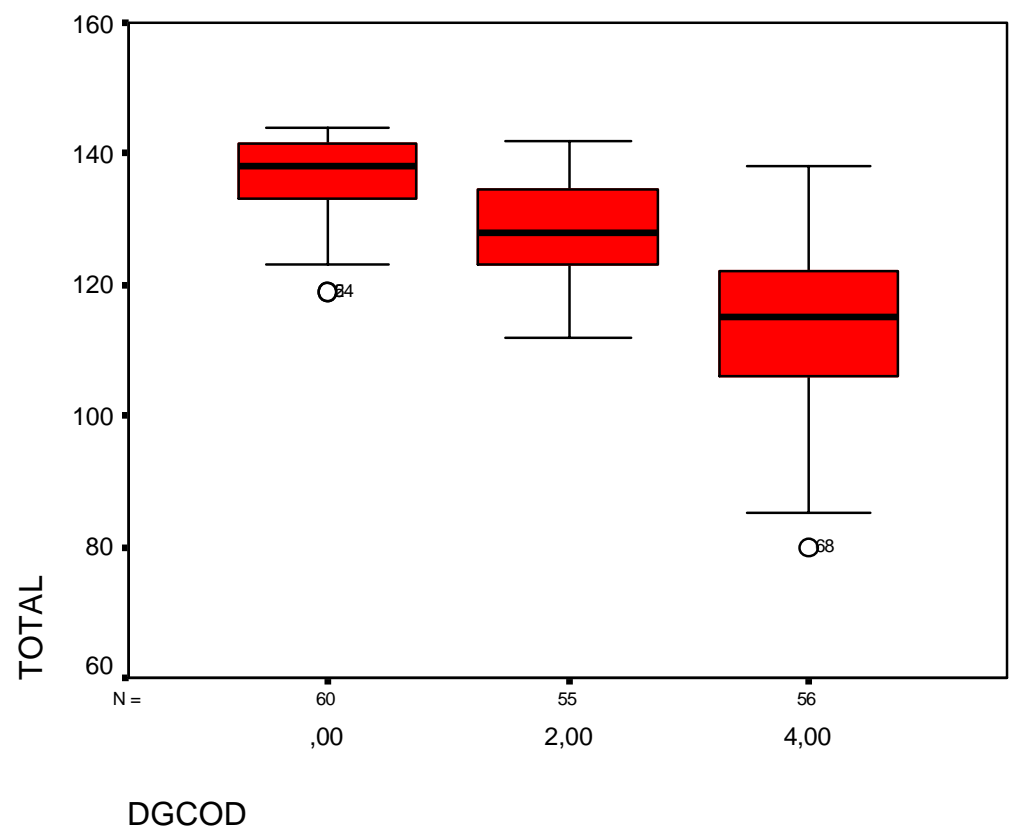

Figura 4: Box plot comparando o desempenho de controles, pacientes com DA e pacientes com CCL no escore total da DRS. DGCOD = 
diagnóstico; $0=$ controles; 1 = DA; $2=$ CCL.

\subsection{Correlação entre as subescalas da DRS e alguns testes neuropsicológicos}

Todas as subescalas da DRS foram correlacionadas a testes neuropsicológicos os quais avaliam funções semelhantes às das subescalas. A subescala Atenção foi correlacionada ao Teste das Trilhas - Parte A, Iniciativa/Perseveração ao teste de fluência verbal para categoria semântica (animais), Construção ao sub-teste Cubos (WAIS), Conceituação ao teste de Matrizes Progressivas de Raven (série colorida), e a subescala Memória foi correlacionada à evocação após 30 minutos do Teste de Aprendizagem Verbal Auditiva de Rey (RAVLT).

Houve muito boa correlação entre a subescala Memória e evocação de 30 minutos do RAVLT $\left(r_{s}=0,752, p=0,001\right)$ e correlação média entre a subescala I/P e fluência verbal $\left(r_{s}=\right.$ 0,588, $\mathrm{p}=0,001)$ e entre a subescala Conceituação e o teste de Raven $\left(\mathrm{r}_{\mathrm{s}}=0,519, \mathrm{p}=0,001\right)$. Entre a subescala Atenção e Teste das Trilhas - Parte A $\left(r_{s}=0,361, p=0,001\right)$ e entre subescala Construção e Cubos $\left(r_{s}=0,314, p=0,001\right)$ não houve uma boa correlação, sendo considerada estatisticamente fraca. 


\section{DISCUSSÃO}

Neste estudo, a DRS apresentou boa acurácia no diagnóstico diferencial entre pacientes com DA de intensidade leve e indivíduos controles, pacientes com DA e pacientes com CCL, e entre pacientes com CCL e indivíduos controles.

A DRS é um instrumento que fornece um maior número de informações a respeito do paciente além de permitir a discriminação entre funções preservadas e comprometidas. A presença de tarefas com grau de dificuldade maior ou menor parece estimular o paciente em continuar a prova além de possibilitar a percepção de suas dificuldades mas também de suas capacidades. O fato de responder corretamente as questões mais difíceis permite que o examinador credite as tarefas subseqüentes, de cada subescala, como corretas e, como conseqüência, o tempo de testagem é diminuído preservando indivíduos relativamente intactos. 
A capacidade de discriminar pacientes com DA de intensidade leve e indivíduos controles, independentemente dos fatores idade e escolaridade já havia sido constatado em estudo anterior (Porto et al., 2003). No presente estudo, as subescalas Memória e I/P foram as que apresentaram maior capacidade de discriminação, em todas as condições analisadas, demonstrando maior acurácia diagnóstica em relação às demais subescalas. A importância das duas subescalas também foi descrita por Monsch et al. (1995) e Chan et al. (2003) que sugeriram uma versão abreviada da DRS somente com estas duas subescalas. Salmon et al. (2002) referem que medidas cognitivas que são efetivas em discriminar entre pacientes com DA de intensidade leve e controles podem ser úteis para detectar pacientes com demência muito leve.

\subsection{Pacientes com DA versus controles}

No diagnóstico diferencial entre DA de intensidade leve e controles, a nota de corte de $<128$, para o escore total da DRS, apresentou sensibilidade de 90,0\% e especificidade de $89,3 \%$.

Em nosso estudo anterior, a nota de corte encontrada foi de $<123$ com sensibilidade de 91,7\% e especificidade de $87,8 \%$ (Porto et al., 2003). A diferença entre as notas de corte se deve, provavelmente, às diferenças demográficas das duas amostras. A média de escolaridade da amostra atual é mais alta, tanto nos pacientes com DA (média $=9,62 \pm 4,68$ ) como nos controles (média $=10,19 \pm 4,73$ ), em relação ao estudo anterior, em que estas foram 9,07 \pm 5,31 e 8,05 \pm 4,62, respectivamente (Porto et al., 2003). Vários estudos ressaltam os efeitos da escolaridade no desempenho da DRS, particularmente no escore total (Bennett et al., 1997, Lucas et al., 1998, Bank et al., 2000, Chan et al., 2001, Foss et al., 2005). Quando comparamos as médias ( e desvios-padrão) do escore total na DRS entre os pacientes e entre os controles nos dois estudos, 
verificamos que foi de 109,68 $\pm 12,03$ e 113,82 $\pm 12,41$ nos pacientes, e $132,55 \pm 9,47$ e $136,21 \pm 6,31$ nos controles sugerindo que a alta escolaridade, da amostra atual, influenciou nos resultados.

As subescalas Memória e I/P foram as que apresentaram melhor poder discriminatório quando se analisou a acurácia, de cada subescala, através das áreas sob as curvas. A subescala Memória contém tarefas de evocação tardia enquanto que $75 \%$ do escore total da subescala I/P se deve ao teste de fluência verbal por categoria. Comprometimento de memória episódica e fluência verbal (memória semântica) são reconhecidamente associados aos primeiros estágios de DA, além de serem consideradas as medidas mais sensíveis para diferenciar idosos não demenciados de pacientes com DA de intensidade leve (Monsch et al., 1992, Weintraub, 2000, Salmon et al., 2002, Arnáiz e Almkvist, 2003,), justificando o fenômeno constatado através das curvas ROC.

A subescala Conceituação demonstrou boa acurácia diagnóstica na discriminação entre pacientes com DA e controles, embora menor que as subescalas Memória e I/P.

Porto et al. (2003) constataram uma dissociação, no escore da subescala Conceituação, entre idosos normais e pacientes com DA de intensidade leve, em função da escolaridade o que permitiu aventar que o comprometimento de abstração e o comprometimento de memória semântica é mais intenso nas fases iniciais de DA em indivíduos de escolaridade abaixo de 11 anos. Bennett et al. (1997) sugerem cautela ao presumir a presença de declínio patológico em idosos institucionalizados com idade acima de 80 anos quando os escores na subescala Conceituação são baixos, particularmente se o nível educacional for inferior a 13 anos de escolaridade. Arnáiz e Almkvist (2003) referem que além de memória episódica verbal e visual e 
fluência verbal também a memória semântica está comprometida em pacientes com DA de intensidade leve. A tarefa sugerida, pelos autores, para avaliar memória semântica foi o sub-teste Semelhanças (WAIS) que contém testes de abstração análogos aos da subescala Conceituação.

A subescala Atenção é composta de tarefas de repetição de dígitos (ordem direta e inversa), atenção concentrada, resposta a dois comandos verbais, leitura de lista com quatro palavras e combinação de desenhos. Em nosso estudo, embora com baixa acurácia, foi capaz de diferenciar os dois grupos estudados. Kipps e Hodges (2005) referem que lapsos em atenção e concentração são comuns e acentuam-se com a idade sendo muito mais evidentes para os pacientes que para os membros da família e, isolados, não são causas de grande preocupação.

A subescala Construção não demonstrou boa acurácia e, portanto não foi capaz de discriminar entre pacientes com DA e controles, de acordo com a afirmação de Vitalino et al. (1984) e Bennett et al. (1997) que consideram os itens desta subescala muito fáceis.

\subsection{Pacientes com DA versus $C C L$}

$\mathrm{Na}$ análise entre pacientes com DA de intensidade leve e pacientes com CCL, a nota de corte de $<123$, para o escore total, apresentou sensibilidade de 78,2\% e especificidade de $76,8 \%$. Em nosso estudo, tentamos classificar pacientes com DA e CCL através de avaliação neuropsicológica extensa, e os dois neurologistas responsáveis pelo diagnóstico dos pacientes basearam-se em resultados de testes neuropsicológicos considerados sensíveis para avaliação de demência. Ganguli et al. (2004) afirmam que o conceito de CCL tem motivado controvérsia entre autores que sugerem que CCL é uma DA incipiente ou leve (Petersen et al., 2001, Morris et al., 2001) e outros que consideram CCL como uma condição instável com validade preditiva pobre 
para DA (Ritchie et al.,2001). Aspectos neuropsicológicos para classificação de CCL não estão bem estabelecidos tanto que não existem evidências suficientes para recomendar testes específicos ou escores de corte específicos (Winblad et al., 2004, Portet et al., 2006). Embora os critérios para classificação dos pacientes tenham sido rígidos em nosso estudo, acreditamos que, muito provavelmente, existe um continuum entre pacientes com CCL e pacientes com DA de intensidade muito leve o que torna a disfunção entre esses dois grupos diagnósticos extremamente difícil. Assim, tanto a sensibilidade como a especificidade encontrada neste estudo, embora inferiores a $80 \%$, podem ser consideradas altas para tão difícil discriminação.

Na análise entre pacientes com DA e CCL, a subescala Memória apresentou melhor acurácia em relação às outras subescalas. Segundo Boeve et al. (2003), pacientes com CCL apresentam escores intermediários, em tarefas de memória, entre sujeitos normais e pacientes com demência, ou mais semelhantes a pacientes com demência. Mesmo assim, em nosso estudo, a subescala Memória apresentou boa acurácia na distinção entre DA leve e CCL.

As subescalas I/P, Conceituação e Atenção foram capazes de discriminar os dois grupos. Como já mencionado, a tarefa de fluência verbal para categoria semântica é responsável por mais de $50 \%$ do escore total da subescala I/P enquanto que a subescala Conceituação contém provas de abstração verbal e visual. As duas subescalas, portanto, contém itens de memória semântica. Feldman e Jacova (2005) afirmam que o processo diagnóstico de CCL envolve avaliação de múltiplos domínios cognitivos, com particular atenção para memória episódica e semântica. Arnáiz e Almkvist (2003) referem que além de tarefas de memória episódica também provas de memória semântica estariam associadas ao aumento do risco de diagnóstico subseqüente de DA. Na comparação do desempenho médio, nas subescalas I/P e Conceituação, entre pacientes com DA e CCL, pacientes com CCL apresentaram resultados significativamente 
melhores. Através de nosso estudo podemos inferir que pacientes com DA de intensidade leve apresentam maior comprometimento de memória semântica confirmando os achados de Porto et al (2003) que referem maior comprometimento de memória semântica em pacientes com DA de intensidade leve na comparação com sujeitos controles. Um fator importante a ser considerado é que, em nossa amostra de CCL, incluímos além de pacientes com CCL amnéstico, pacientes com CCL de múltiplos domínios o que pode ter contribuído para os resultados encontrados.

A subescala Atenção, entre pacientes com DA e CCL apresentou uma acurácia diagnóstica muito próxima a encontrada entre pacientes com DA e controles, diferenciando pacientes com DA de CCL. No entanto, na observação das médias entre os dois grupos observamos desempenho muito próximo, com mediana igual a 35 para DA e 36 para CCL. Podemos inferir baseados nos achados de nosso estudo, que déficits atencionais ocorrem em pacientes com DA leve o suficiente para distinguir estes pacientes com CCL, mas não de forma tão significativa.

A baixa acurácia da subescala Construção também foi observada entre pacientes com DA e CCL.

\subsection{Pacientes com $\mathrm{CCL}$ versus controles}

Na distinção entre pacientes com CCL e controles, a nota de corte de <134 demonstrou sensibilidade de $73,3 \%$ e $72,2 \%$ de especificidade. A baixa sensibilidade e especificidade, do escore total da DRS, poderia sugerir que esta escala não permite distinguir, de forma acurada, as duas condições analisadas, contrário aos achados de Salmon et al. (2002) que refere excelente 
acurácia da DRS associada a testes de evocação tardia e fluência verbal na discriminação de pacientes com DA muito leve e controles.

A subescala Memória foi a que demonstrou maior área sob a curva ROC demonstrando maior acurácia diagnóstica em relação às outras subescalas. Um dos principais critérios para diagnóstico de CCL amnéstico é a presença de queixa de memória confirmada por baixos escores em tarefas de memória (Winblad et al.,2004) o que explicaria a boa acurácia da subescala Memória na discriminação entre pacientes com CCL e controles.

No estudo de Charchat-Fichman (2003), a subescala Memória foi empregada como um dos critérios de classificação dos pacientes com CCL, de modo que não foi possível verificar qual a acurácia da DRS naquele estudo e estabelecer comparações com este.

Embora a área sob a curva da subescala $\mathrm{I} / \mathrm{P}$ também tenha apresentado valor de significância na diferenciação de CCL e controles, a sensibilidade e especificidade da nota de corte desta subescala foram muito baixas. A média na subescala I/P para pacientes com CCL foi $34,14 \pm 3,42$ e para controles foi $35,95 \pm 1,88$, o que poderia explicar a baixa acurácia da subescala. Para maior compreensão dos achados, estudos mais detalhados, em uma amostra maior, diferenciando pacientes com CCL amnéstico e CCL de múltiplos domínios, se fazem necessário.

As subescalas Atenção, Conceituação e Construção, conforme esperávamos, não diferenciaram os dois grupos. 


\subsection{CCL como um estado intermediário entre idosos normais e pacientes com DA}

leve.

Quando se comparou o desempenho de pacientes com DA, pacientes com CCL e indivíduos controles na DRS constatou-se diferença significativa no escore total e em todas as subescalas. O conceito de CCL é utilizado, em vários estudos, como um estágio intermediário entre a normalidade e demência, sugerindo clinicamente um risco ou um estado prodrômico para doença de Alzheimer (Portet et al., 2006, Ganguli et al., 2004, Arnáiz e Almkvist, 2003, Petersen et al., 2001). De acordo com nossos achados, muito provavelmente a subescala Memória exerceu papel importante no declínio gradativo observado entre os três grupos. Em nosso estudo, a subescala Memória foi a que melhor diferenciou todos os grupos analisados e as notas de corte encontradas, para esta subescala, pode contribuir para o diagnóstico de pacientes com queixa de memória.

\subsection{Correlação entre as subescalas da DRS e alguns testes neuropsicológicos}

Na correlação entre as subescalas da DRS e testes neuropsicológicos que avaliam funções semelhantes às das subescalas, encontramos correlação significativa entre a subescala Memória e o teste de memória episódica, entre a subescala I/P e o teste de fluência verbal de categoria semântica e entre a subescala Conceituação e a teste de pensamento abstrato. 
A melhor correlação constatada foi entre a subescala Memória e o teste de memória episódica (evocação da lista de palavras - RAVLT). O teste de evocação de listas de palavras é sugerido no Consenso do Grupo de Trabalho Internacional sobre CCL, por Winblad et al. (2004), para avaliação de memória episódica em pacientes com CCL. A forte correlação da subescala Memória vem de encontro ao achado da elevada acurácia desta subescala tanto na diferenciação de pacientes com DA versus pacientes com CCL como na diferenciação de pacientes com CCL versus controles.

As subescalas I/P e Conceituação apresentaram uma correlação média com os testes fluência verbal e Raven respectivamente. Embora o teste de fluência verbal seja responsável por 20 pontos dos 37 do escore total da subescala I/P, os itens desta subescala também incluem provas de praxias e cópia de sequiência de figuras o que poderia explicar a correlação não tão elevada. Quanto a subescala Conceituação, o teste de Raven é uma tarefa visual enquanto que os itens da subescala, incluem em sua maioria, testes verbais de abstração, o que poderia explicar a correlação média encontrada.

As subescalas Atenção e Construção apresentaram uma correlação fraca com os testes selecionados (Teste das Trilhas - Parte A e sub-teste Cubos). O Teste das Trilhas - Parte A é uma prova de atenção que envolve exploração visual e rapidez muito diferente dos itens que compõem a subescala Atenção o que justificaria a fraca correlação entre as duas tarefas. No estudo de Boeve et al. (2003), os autores observaram que pacientes com CCL apresentaram escores no Teste das Trilhas - Parte A semelhantes aos dos controles enquanto que os escores da Parte B, do mesmo teste, foram intermediários entre controles e pacientes com demência. 
Quanto a ausência de correlação significativa da subescala Contrução, resultado semelhante foi constatado no estudo realizado por Marson et al. (1997) no qual o subteste Cubos (WAIS) não se correlacionou com a subescala Construção. A subescala Construção contém itens simples de praxias grafo-motoras enquanto que o sub-teste Cubos é uma prova de construção com blocos tridimensional que envolve funções vísuo-contrutivas e vísuo-motoras, acessando processos cognitivos diferentes (Marson et al., 1997).

Finalizando, este estudo reafirmou o valor da DRS, confirmando os achados prévios (Porto et al., 2003), no diagnóstico de pacientes com DA de intensidade leve. A DRS também foi capaz de diferenciar entre pacientes com CCL e pacientes com DA e entre pacientes com CCL e indivíduos controles, embora com menor acurácia do que na distinção entre controles e pacientes com DA de intensidade leve.

Uma das limitações do trabalho foi a ausência da investigação da influência da escolaridade. Como verificado em outros estudos esta variável interfere de maneira significativa no desempenho da DRS. Em nossa amostra, tanto pacientes como controles, apresentaram alta escolaridade o que não permitiu a análise por grupos de escolaridade. Outra limitação foi a inclusão de dois grupos de CCL, amnéstico e de múltiplos domínios, em um único grupo. A análise separada do desempenho destes pacientes, que não foi possível pelo número relativamente pequeno de pacientes que seriam alocados em cada subgrupo, poderá contribuir para melhor compreensão do valor da DRS na avaliação destes pacientes. 


\section{CONCLUSÕES}

A DRS demonstrou ser um instrumento com boa acurácia diagnóstica na discriminação entre pacientes com doença de Alzheimer de intensidade leve e indivíduos controles, confirmando observações realizadas em outra amostra de pacientes e controles. 
A DRS foi capaz de diferenciar pacientes com comprometimento cognitivo leve de sujeitos controle embora com menor acurácia do que na diferenciação entre pacientes com doença de Alzheimer de intensidade leve e sujeitos controles.

A DRS foi capaz de diferenciar pacientes com comprometimento cognitivo leve de pacientes com doença de Alzheimer de intensidade leve embora com menor acurácia do que na distinção entre pacientes com doença de Alzheimer de intensidade leve e controles.

As subescalas Memória e Iniciativa/Perseveração confirmaram observações anteriores como as subescalas que melhor discriminam pacientes com doença de Alzheimer de intensidade leve e indivíduos controles.

A subescala Memória demonstrou maior acurácia em relação às demais subescalas na diferenciação entre pacientes com doença de Alzheimer leve e indivíduos controles, entre pacientes com doença de Alzheimer de intensidade leve e pacientes com comprometimento cognitivo leve, e entre pacientes com comprometimento cognitivo leve e sujeitos controles.

\section{REFERÊNCIAS BIBLIOGRÁFICAS}

American Psychiatric Association. Diagnostic and Statistical Manual of Mental Disorders. 3rd ed. Ver. Washington, DC: American Psychiatric Association, 1987. 
Abrisqueta - Gomez JA. Avaliação Neuropsicológica nas Fases Inicial e Moderada da Demência do Tipo Alzheimer. São Paulo, 1999. Tese (Doutorado). Escola Paulista de Medicina - Universidade Federal de São Paulo.

Arnáiz E, Almkvist O. Neuropsychological features of mild cognitive impaiment and preclinical Alzheimer's disease. Acta Neurol Scand. 2003; 107 (179): 34-41.

Bank AL, Yochim BP, MacNeill SE, Lichtenberg PA. Expanded Normative Data for the Mattis Dementia Rating Scale for Use with Urban, Elderly Medical Patients. The Clinical Neuropsychologist 2000; 14: 149-156.

Bennett A, Nadler J, Spigler M, Rafalson L, Abraham S, Relkin N. The Mattis Dementia Rating Scale in Nursing Home Octagenarians and Nonagenarians: Effects of age and education. Journal of Geriatric Psychiatry and Neurology 1997; 10: 114-118.

Bertolucci PHF, Okamoto IH, Toniolo Neto J, Ramos LR, Brucki SMD. Desempenho da população brasileira na bateria neuropsicológica do Consortium to Establish a Registry for Alzheimer's Disease (CERAD). Revista de Psiquiatria Clínica 1998; 25 (2): 88-97.

Bertolucci PHF, Brucki MD, Campacci R, Juliano Y. O Mini-Exame do Estado Mental em uma População Geral. Impacto da escolaridade. Arquivos de Neuro-Psiquiatria. 1994; $52(1): 1-7$.

Bustamante SEZ, Bottino CMC, Lopes MA, Azevedo D, Hototian SR, Litvoc J, Jacob Filho W. Instrumentos combinados na avaliação de demência de idosos. Arq Neuropsiquiatr. 2003; 61(3-A): 601-606).

Boeve B, McCormick J, Smith G, Ferman T, Rummans T, Carpenter T, Ivnik R, Kokmen E, et al. Mild cognitive impairment in the oldest old. Neurology. 2003; 60: 477-480.

Bottino CMC; Stoppe Jr A, Scalco AZ, Ferreira RCR, Hototian SR, Scalco MZ. Validade e confiabilidade da versão brasileira do CAMDEX. Arquivos de Neuro-Psiquiatria 2001; 59 (Suppl 3): 20. Resumo. 
Brucki,SMD; Nitrini, R; Bertolucci, PHP; Caramelli, P; Okamoto, IH. Normas sugeridas para o uso do Mini-Exame do Estado Mental (MEEM) em nosso meio. Arq Neuropsiquiatr 2003; 60: 46-47.

Chan AS, Choi A, Chiu H, Lam L. Clinical validity of the Chinese version of Mattis Dementia Rating Scale in differentiating dementia os Alzheimer's type in Hong Kong. $J$ Int Neuropsychol Soc. 2003; 9 (1): 45 -55.

Chan AS, Choi MK, Salmon DP. The effects of age, education, and gender on the Mattis Dementia Rating Scale performance of elderly Chinese and American individuals. $J$ Gerontol B Psychol Sci Soc Sci. 2001; 56 (6): 356-63.

Charchat-Fichman H. Heterogeneidade neuropsicológica no processo de envelhecimento: transição do normal aos estágios da Doença de Alzheimer. Tese (Doutorado). São Paulo: Instituto de Psicologia, Universidade de São Paulo; 2003.

Coblentz JM, Mattis S, Zingesser LH, Kasoff SS, Wisniewski HM, Katzman R. Presenile Dementia. Clinical Aspects and Evaluation of Cerebrospinal Fluid Dynamics. Arch Neurol.1973; 29: 299-308.

Diniz, LFM; Cruz, MF; Torres, VM; Consenza, RM. O teste de aprendizagem auditivoverbal de Rey: normas para uma população brasileira. Rev bras Neurol 2000; 36 (3): 7983.

Fama R, Sullivan EV, Shear PK, Marsh L, Yesavage JA, Tinklenberg JR, Lim KO, Pfefferbaum A. Selective cortical and hipocampal volume correlates of Mattis Dementia Rating Scale in Alzheimer disease. Arch Neurol. 1997; 54:719- 728.

Feldman HH, Jacova C. Mild cognitive impairment. Am J Geriatr Psychiatry. 2005; 13 (8): 645-55.

Folstein MF, Folstein SE, McHugh PR. "Mini-mental state". A practical method for grading the cognitive state of patients for the clinician. J. Psychiatr Research. 1975;12: 189-198. 
Foss MP, Valle Fde AC, Speciali JG. Influência da escolaridade na avaliação neuropsicológica de idosos: aplicação e análise dos resultados da Escala de Mattis para Avaliação de Demência (Mattis Dementia Rating Scale - MDRS). Arq Neuropsiquiatr. 2005; 63 (1): 119-26.

Ganguli M, Dogge HH, Shen C, DeKosky ST. Mild cognitive impaiment, amnestic type: an epidemiologic study. Neurology. 2004; 63: 115-121.

Geda Ye, Smith GE, Knopman DS, Boeve BF, Tangalos EG, Ivnik RJ, Mrazek DA, Edland SC, petersen RC. International Psychogeriatrics. 2004; 16 (1): 51-60.

Griffith HR, Netson KL, Harrell LE, Zamrini EY, Brockington JC, Marson DC.

Amnestic mild cognitive impairment: diagnostic outcomes and clinical prediction over a two-year time period. J Int Neuropsychol Soc. 2006; 12(2): 166-75.

Griffith HR, Martin RC, Bambara JK, Marson DC, Faught E. Older adults with epilepsy demonstrate cognitive impairments compared with patients with amnestic mild cognitive impairment. Epilepsy Behav. 2006; 8 (1):161-8.

Goodglass H, Kaplan E. The assessment of aphasia and related disorders. $2^{\text {nd }}$ ed. Philadelphia: Lea \& Febiger, 1987.

Herrera Junior E, Caramelli P, Nitrini R. Estudo epidemiológico populacional de demência na cidade de Catanduva - estado de São Paulo - Brasil. Revista de Psiquiatria Clínica 1998; 25 (2): 70 - 73.

Hoffer SM, Piccinin AM, Hershey D. Analysis of structure and discriminative power of the Mattis Dementia Rating Scale. Journal of Clinical Psychology 1996; 52: 395-409.

Hooper Visual Organization Test (VOT) Manual. Western Psychological Services. 1983.

Jorm AF. The Epidemiology of Alzheimer's Disease and Related Disorders. London: Chapaman and Hill,1990. 
Jorm, AF. A short-form of the Informant Questionnaire on Cognitive Decline in the Elderly (IQCODE): development and cross-validation. Psychological Medicine 1994; 24 : 145-153.

Karbe H, Kertesz A, Davis J, Kemp BJ, Prato FS, Nicholson RL. Quantification of functional deficit in Alzheimer's disease using a computer-assisted mapping program for Tc-HMPAO SPECT. Neuroradiology 1994; 36: 1-6.

Kertesz A, Clydesdale S. Neuropsychological Deficits in Vascular Dementia vs Alzheimer's Disease. Arch Neurol 1994; 51:1226-1231.

Kipps CM, Hodges JR. Cognitive assessment for clinicians. J Neurol Neurosurg Psychiatry. 2005; 76: 22-30

Knox MR, Lacritz LH, Chandler MJ, Munro Cullum C. Association between Dementia Rating Scale performance and neurocognitive domains in Alzheimer's disease. Clin Neuropsychol. 2003 May; 17 (2): 216 - 9.

Lezak, MD. Neuropsychological Assessment. 3a Edição. New York. Oxford University Press, 1995.

Lucas JA, Ivnik RJ, Smith GE, Bohac DL, Tangalos EG, Kokmen E, Graff-Radford NR, Petersen RC. Normative Data for the Mattis Dementia Rating Scale. Journal of Clinical and Experimental Neuropsycholy 1998; 20 (4): 536-547.

Lukatela K, Cohen R, Kessler H, Jenkins MA, Moser DJ, Stone WF, Gordon N, Kaplan RF. Dementia Rating Scale Performance: A Comparison of Vascular and Alzheimer's Dementia. Journal of Clinical and Experimental Neuropsycholy 2000; 22(4):445-454.

Marson DC, Dymek MP, Duke LW, Harrell LE. Subscale Validity of the Mattis Dementia Rating Scale. Archives of Clinical Neuropsychology 1997; 12(3):269-275.

Mattos, P; Lino, V; Rizo, L; Alfano, A; et al. Memory complaints and test performance in health elderly persons. Arq Neuropsiquiatr 2003; 61 (4): 920-924. 
Mattis S. Mental Status Examination for Organic Mental Syndrome in the Elderly Patient. In Bellak L, Karasu TB, eds Geriatric Psychiatry. A Handbook for Psychiatrists and Primary Care Physicians. New York. Grune \& Stratton, :1976: 77-121.

Mattis S. Dementia Rating Scale. Professional Manual. Florida: Psychological Assessment Resources, Inc, 1988.

McKhann G; Drachman D; Folstein M; Katzman R; Price D; Stadlan EM. Clinical Diagnosis of Alzheimer's disease: report of the NINCDS-ADRDA work group under the auspices of department of health and human services task force on Alzheimer's disease. Neurology 1984; 34: 939-944.

Mesulam M-M. Principles of Behavioral Neurology. F A Davis Company, 1985.

Mesulam M-M. Principles of Behavioral and Cognitive Neurology. Second Edition. Oxford University Press, 2000.

Miller JM, Pliskin NH. The clinical utility of the Mattis Dementia Rating Scale in assessing cognitive decline in Alzheimer's diasease. Int J Neurosci. 2006 May; 116 (5): 613-27.

Monsch AU, Bondi MW, Butters N, Salmon DP, Katzman R, Thal LJ. Comparisons of verbal fluency tasks in the detection of dementia of the Alzheimer type. Arch Neurol 1992; 49: 1253-1258.

Monsch AU, Bondi MW, Salmon DP, Butters N, Thal LJ, Hansen LA, Wiederholt WC, Cahn DA, Klaubert MR. Clinical Validity of the Mattis Dementia Rating Scale in Detecting Dementia of the Alzheimer Type. Arch Neurol. 1995; 52: 899-904.

Morris JC, Heyman A, Mohs RC, Hughes JP, van Belle G, Fillenbaum G, Mellits ED, Clark C. The Consortium to Establish a Registry for Alzheimer's Disease (CERAD). Part I. Clinical and neuropsychological assessment of Alzheimer's disease. Neurology 1989; 39: 1159-1165. 
Morris JC, Storandt M, Miller JP, et al. Mild cognitive impairment represents early stage Alzheimer disease. Arch Neurol. 2001; 58: 397-405

Nitrini R, Lefévre BH, Mathias SC, Caramelli P, Carrilho PE, Sauaia N, Massad E, Takiguti C, Da Silva IO, Porto CS, et al. Neuropsychological tests of simple application for diagnosing dementia. Arq Neuropsiquiatr. 1994; 52(4): 457-65.

Nitrini R, Caramelli P, Porto CS, Charchat-Fichman H, Formigoni AP Otero C, Prandini JC. Avaliação Cognitiva Breve no diagnóstico de doença de Alzheimer leve. Arq Neuropsiquiatr 2005; 63 (2): 27.

Nitrini R. Demências. In: Geriatria, Fundamentos, Clínica e Terapêutica. Carvalho Filho ET, Papaléo Netto M. $2^{\mathrm{a}}$ edição. Atheneu, São Paulo, 2005.

Ostrosky-Solis F, Ardila A, Rosselli M. NEUROPSI: A brief neuropsychological test battery in spanish with norms by age and educacional level. JINS 1999; 5 (5): 413-433.

Paul RH, Cohen RA, Moser D, Ott BR, Zawacki T, Gordon N, Bell S, Stone W. Performance on the Mattis Dementia Rating Scale in patients with vascular dementia: relationships to neuroimaging findings. J Geriatr Psychiatry Neurol. 2001; 14 (1): 33-6.

Paulsen JS, Butters N, Sadek JR, Johnson AS, Salmon DP, Swerdlow NR, Swenson MR. Distinct cognitive profiles of cortical and subcortical dementia in advanced illness. Neurology 1995; 45(5): 951-956.

Paolo AM, Trostr AI, Glatt SL, Hubble JP, Koller WC. J. Differentiation of the dementias of Alzheimer's and Parkinson's disease with the dementia rating scale. Geriatr Psychiatry Neurol. 1995; 8 (30): 184-188.

Petersen RC, Smith GE, Waring SC, Ivnik RJ, Tangalos EG, Kokmen E. Mild cognitive impairment. Clinical caracterization and outcome. Arch Neurol. 1999; 56: 303-308.

Petersen, RC; Stevens, JC; Ganguli, M; Tangalos, EG; et al. Practice parameter: Early detection of dementia: Mild cognitive impairment (an evidence-based review). Report of 
the Quality Standars Subcommittee of the American Academy of Neurology. Neurology 2001; 56: 1133-1142.

Pfeffer, RI; Kusosaki, TT; Harrah Jr, CH; Chance JM; Filos, S. Measurement of Functional Activities in Older Adults in the Community. Journal of Gerontology 1982; 37 (3): 323- 329.

Portet F, Ousset PJ, Visser PJ, Frisoni GB, Nobili J, Scheltens Ph, Vellas B, Touchon J. Mild cognitive impairment in medical practice: critical review of the concept and new diagnostic procedure. Report of the MCI working group of the European Consourtium on Alzheimer's disease (EADC). J Neurol Neurosurg Psychiatry published online. 2006. doi:10.1136/jnnp2005.085332

Porto, CS. A escala de avaliação de demência (DRS) no diagnóstico de demência de Alzheimer. São Paulo, 2002. Dissertação (Mestrado). Universidade de São Paulo.

Porto, CS; Charchat-Fichman,H; Caramelli, P; Bahia, VS; Nitrini,R. Brazilian version of the Mattis Dementia Rating Scale. Diagnosis of mild dementia in Alzheimer's disease. Arq Neuropsiquiatr 2003; 61 (2-B): 339-345.

Radanovic, M; Mansur, LL; Scaff, M. Normative data for the Brazilian population in the Boston Diagnostic Aphasia Examination: influence of schooling. Braz. J. Med. Biol. Res 2004; 37 (11): 1731-1738.

Raven, JC; Raven J; Court, JH. Manual Matrizes Progressivas Coloridas. Casa do Psicólogo. Divisão Editorial. 1988.

Rey A. Figuras Complexas de Rey. Casa do Psicólogo, 1998.

Rilling LM, Lucas JÁ, Ivnik RJ, Smith GE, Willis FB, Ferman TJ, Petersen RC, GraffRadford NR. Mayo's Older African American Normative studies: norms for the Mattis Dementia Rating Scale. Clin neuropsychol. 2005; 19 (2): 229-42. 
Ritchie K, Artero S, Touchon J. Classification criteria for mild cognitive impairment: a population-based validation study. Neurology. 2001; 56: 37-42.

Rosen WG; Mohs RC; Davis KL. A new rating scale for Alzheimer's disease. Am J. Psychiatry 1984; 141: 1356-1364.

Roth M; Tym E; Mountjoy CQ; Huppert FA; Hendrie H; Verma S; Goddard S. CAMDEX: a standartised instrument for the diagnosis of mental disorder in the elderly with special reference to the early detection of dementia. Br J Psychiatry. 1986; 698-709.

Salmon DP, Thal LJ, Butters N, Heindel WC. Longitudinal evaluation of dementia of the Alzheimer type: A comparision of 3 standardized mental status examinations. Neurology 1990; 40: 1225-1230.

Salmon DP, Thomas RG, Pay MM, Booth A, Hofstetter CR, Thal LJ, Katzman R. Alzheimer's disease can be accurately diagnosed in very midly impaired individuals. Neurology. 2002; 59: 1022-1028.

Schmidt R, Freidl W, Fazekas F, Reinhart B, Grieshofer P, Koch M, Eber B, Schumacher M, Polmin K, Lechner H. The Mattis Dementia Rating Scale: Normative data from 1,001 healthy volunteers. Neurology 1994; 44: 964-966.

Schultz RR. Desempenho de uma população brasileira na subescala cognitiva da "Escala de Avaliação da Doença de Alzheimer" (ADAS-Cog). ). São Paulo, 1999. Dissertação (Mestrado). Escola Paulista de Medicina - Universidade Federal de São Paulo.

Spreen O and Strauss E. A Compendium of Neuropsychological Tests. Administration, Norms, and Commentary. Second Edition. Oxford University Press, 1998.

Stout JC, Jernigan TL, Archibald SL, Salmon DP. Association of Dementia Severity With Cortical Gray Matter and Abnormal White Matter Volumes in Dementia of the Alzheimer Type. Arch Neurol. 1996; 53:742-749. 
Vanotti S, Yorio A, Atlasovich C, Labos E. Normatización de la Escala de Graduación de Demencia de Mattis para la población rioplantense. Revista Latina de Pensamiento y Lenguage 2001; 111. Resumo.

Vitalino PP, Breen AR, Russo J, Albert M, Vitiello MV, Prinz PN. J. The clinical utility of the dementia rating scale for assessing Alzheimer patients. J Chron Dis 1984; 37 (9/10):743-753.

Xavier FMF, Ferraz MPT, Trentini CM, Freitas NK, Moriguchi EH. Bereavement-related cognitive impairment in a oldest-old community-dwelling brazilian sample. Journal of Clinical and Experimental Neuropsychology. 2002, 24(2): 294-301.

Wechsler D. A standardized memory scale for clinical use. Journal of Psychology 1945; 19:87-95.

Wechsler D. Wechsler Memory Scale. Manual The Psychological Corporation Harcourt Brace Jovanovich, 1987.

Wechsler, D. Test de Inteligencia para adultos (WAIS). Manual. 2a Edição. Buenos Aires, Argentina. Editorial Paidos,1993.

Weintraub S. Neuropsychological assessment of mental state. In: Principles of Behavioral and Cognitive Neurology. $2^{\mathrm{a}}$ edition. Oxford. 2000.

Welsh KAB, Mohs RC. Neuropsychological assessment of Alzheimer's disease. Neurology 1997; 49 (Suppl 3): S11-S13.

Winblad B, Palmer K, Kivipelto M, Jelic V, Fratiglioni L, Wahlund LO, et al. Mild cognitive impaiment - beyond controversies, towards a consensus: report of the International Working Group on Mild Cognitive Impairment. Jounal of Internal Medicine. 2004; 256: 240-246. 
APÊNDICE 


\section{Mem. Incidental Mem. Imediata Aprendizado Mem. Tardia Reconh.}

Sapato

Casa

Pente

Chave

Avião

Balde

Tartaruga

Livro

Colher

Árvore

Escores (Corretas)
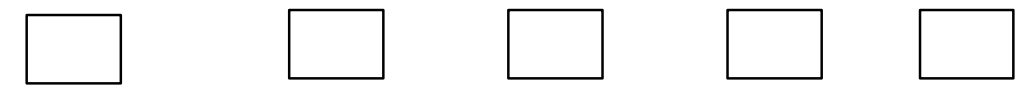

Intrusões
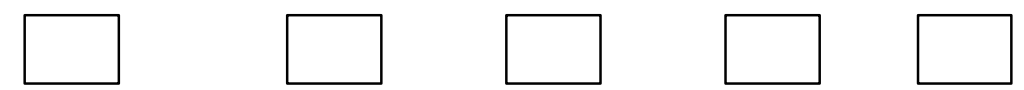

Atenção: Para o Reconhecimento, o escore final é obtido pela subtração: corretas menos intrusões.

Reconhecimento: 


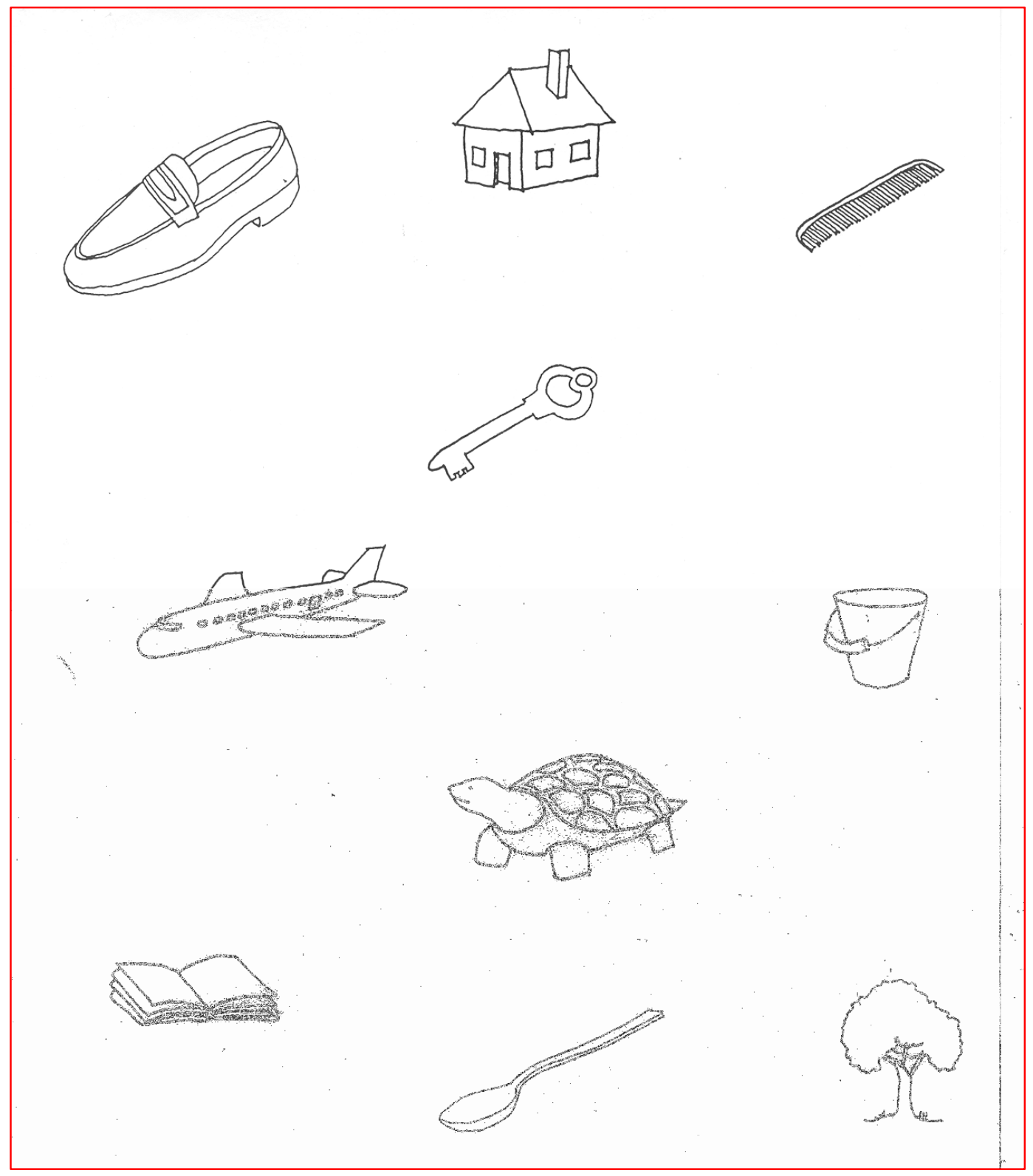




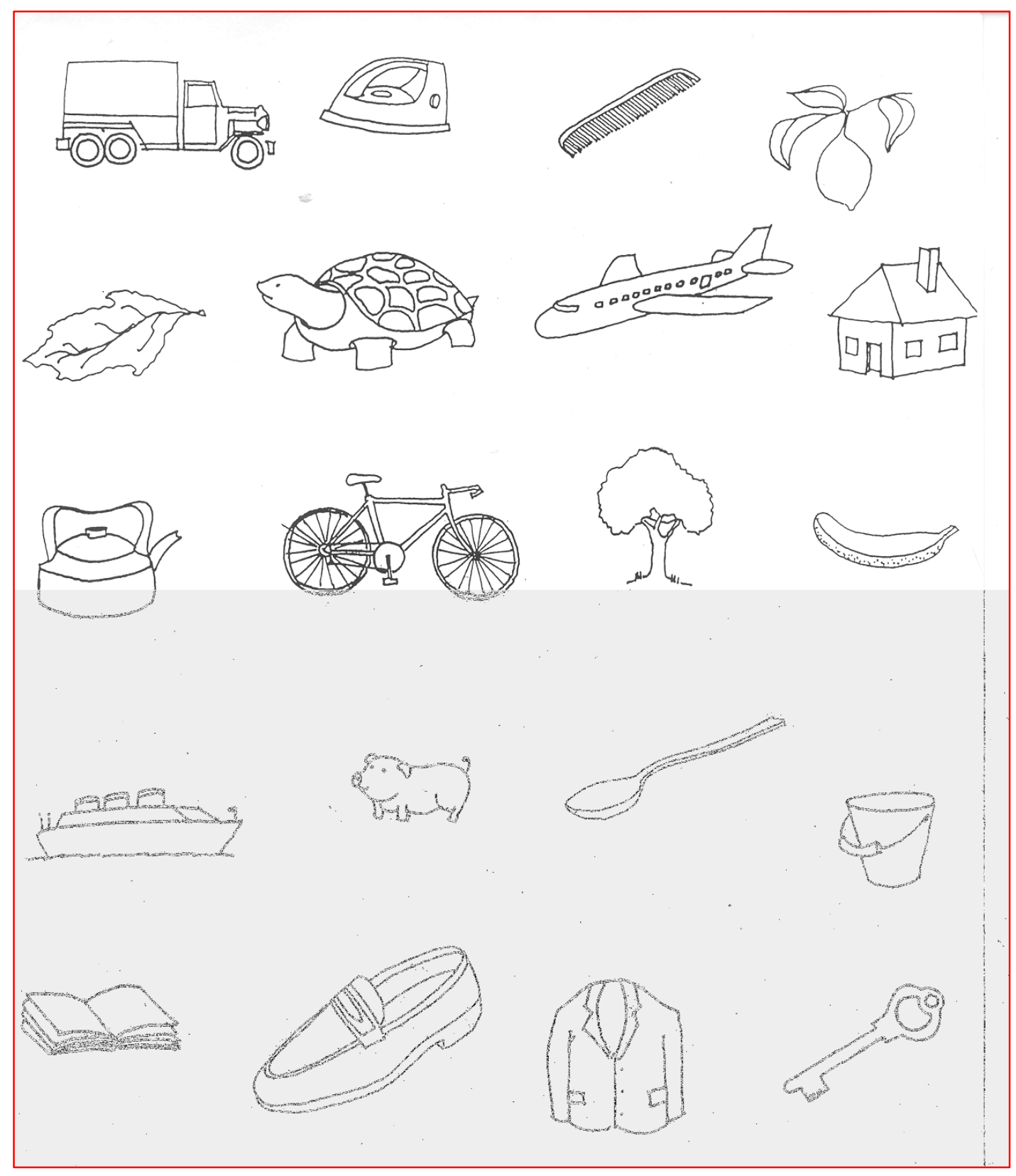




\section{Informant Questionnaire on Cognitive Decline in the Elderly- (Jorm, 1994)}

Agora, nós queremos que você se lembre como o seu amigo ou familiar estava há 10 anos atrás e compare com o estado em que ele está agora. 10 anos era 1993. Abaixo, são situações em que esta pessoa usa sua memória ou inteligência e nós queremos que você indique se estas situações melhoraram, permaneceram do mesmo jeito ou se pioraram nos últimos 10 anos. Observe a importância de comparar a sua performance presente com a de 10 anos atrás. Então, se há 10 anos atrás esta pessoa sempre se esquecia onde ela guardava as coisas, e ela ainda se esquece, então isto seria considerado "não muito alterado". Por favor, indique as alterações que você observou, marcando com um "X" na resposta apropriada.

\begin{tabular}{|c|c|c|c|c|}
\hline Comparada há 10 anos atrás, como está a pessoa nas situações: & $\begin{array}{l}\text { Muito } \\
\text { melhor }\end{array}$ & $\begin{array}{ll}\text { Um pouco } & \text { Não muito } \\
\text { melhor } & \text { alterado }\end{array}$ & $\begin{array}{l}\text { Um } \\
\text { pouco } \\
\text { pior }\end{array}$ & $\begin{array}{l}\text { Muito } \\
\text { pior }\end{array}$ \\
\hline \multicolumn{5}{|l|}{$\begin{array}{l}\text { 1. Lembrar-se de coisas sobre a família e amigos, p. ex. } \\
\text { ocupações, aniversários, endereços }\end{array}$} \\
\hline \multicolumn{5}{|l|}{ 2. Lembrar-se de coisas que aconteceram recentemente } \\
\hline \multicolumn{5}{|l|}{ 3. Lembrar-se do que conversou nos últimos dias } \\
\hline \multicolumn{5}{|l|}{ 4. Lembrar-se de seu endereço e telefone } \\
\hline \multicolumn{5}{|l|}{ 5. Lembrar-se do dia e mês correntes } \\
\hline \multicolumn{5}{|l|}{ 6. Lembrar-se onde as coisas são guardadas usualmente } \\
\hline \multicolumn{5}{|l|}{$\begin{array}{l}\text { 7. Lembrar-se onde foram guardadas coisas que foram } \\
\text { colocadas em locais diferentes do usual }\end{array}$} \\
\hline \multicolumn{5}{|l|}{ 8. Saber como os aparelhos da casa funcionam } \\
\hline \multicolumn{5}{|l|}{ 9. Aprender como usar novos aparelhos da casa } \\
\hline \multicolumn{5}{|l|}{ 10. Aprender coisas novas em geral } \\
\hline \multicolumn{5}{|l|}{ 11. Acompanhar uma história em um livro ou na televisão } \\
\hline \multicolumn{5}{|l|}{ 12. Tomar decisões em problemas do dia-a-dia } \\
\hline \multicolumn{5}{|l|}{ 13. Manusear dinheiro para as compras } \\
\hline \multicolumn{5}{|l|}{$\begin{array}{l}\text { 14. Lidar com problemas financeiros, como por exemplo, } \\
\text { pensão, coisas de banco }\end{array}$} \\
\hline $\begin{array}{l}\text { 15. Lidar com outros problemas matemáticos do dia-a-dia, com } \\
\text { opor exemplo, saber quanta comida comprar, saber quanto } \\
\text { tempo transcorreu entre as visitas de familiares e amigos } \\
\text { 16.Usar sua inteligência para entender qual o sentido das coisas }\end{array}$ & & & & \\
\hline & & & & \\
\hline
\end{tabular}




\section{$M A C-Q$}

Versão Brasileira (adaptada por Xavier ): Questionário de Queixas Subjetivas de

Memória (MAC-Q). "Comparado com: "Como o Sr. (a) era aos 40 anos, como o Sr. (a) descreveria a sua capacidade para realizar as seguintes tarefas que envolvem a memória:

\begin{tabular}{|c|c|c|c|c|c|}
\hline & $\begin{array}{l}\text { Muito melhor } \\
\text { agora (1) }\end{array}$ & $\begin{array}{c}\text { Um pouco } \\
\text { melhor agora (2) }\end{array}$ & $\begin{array}{c}\text { Sem } \\
\text { mudança (3) }\end{array}$ & $\begin{array}{l}\text { Um pouco pior } \\
\text { agora (4) }\end{array}$ & $\begin{array}{l}\text { Muito pior } \\
\text { agora (5) }\end{array}$ \\
\hline $\begin{array}{l}\text { 1.Lembrar o } \\
\text { nome de pessoas } \\
\text { que acabou de } \\
\text { conhecer }\end{array}$ & & & & & \\
\hline $\begin{array}{l}\text { 2.Lembrar o } \\
\text { número do } \\
\text { telefone que usa } \\
\text { pelo menos uma } \\
\text { vez por semana }\end{array}$ & & & & & \\
\hline $\begin{array}{l}\text { 3.Lembrar onde } \\
\text { colocou objetos } \\
\text { (ex. chaves) }\end{array}$ & & & & & \\
\hline $\begin{array}{l}\text { 4.Lembrar } \\
\text { notícias de uma } \\
\text { revista ou da } \\
\text { televisão }\end{array}$ & & & & & \\
\hline $\begin{array}{l}\text { 5.Lembrar coisas } \\
\text { que pretendia } \\
\text { comprar quando } \\
\text { chega ao local }\end{array}$ & & & & & \\
\hline $\begin{array}{l}\text { 6.Em geral, como } \\
\text { descreveria sua } \\
\text { memória } \\
\text { comparada aos } \\
40 \text { anos de idade? }\end{array}$ & & & & & \\
\hline
\end{tabular}

OBS:as cinco primeiras perguntas são pontuadas da seguinte forma: muito melhor agora (1 ponto); um pouco melhor agora ( 2 pontos); sem mudança ( 3 pontos); um pouco pior agora ( 4 pontos); muito pior agora (5 pontos). Já a pergunta seis é pontuada da seguinte forma: muito melhor agora ( 2 pontos); um pouco melhor agora (4 pontos); sem mudança (6 pontos); um pouco pior agora ( 8 pontos); muito pior agora (10 pontos).

A pontuação no teste pode variar de 7 pontos até 35, sendo a pontuação máxima relacionada com Percepção subjetiva de disfunção na memória. 
QUESTIONÁRIO DE ATIVIDADES FUNCIONAIS (Pfeffer, 1982)

1) Ele (Ela) manuseia seu próprio dinheiro?

$\square$ normal
$\square$ faz com dificuldade
$\square$ necessita de ajuda

$\square$ não é capaz $\square$ nunca o fez, mas poderia fazê-lo agora

$\square$ nunca o fez e agora teria dificuldade

2) Ele (Ela) é capaz de comprar roupas, comida, coisas para casa sozinho (a)?

$\square$ normal

$\square$ faz com dificuldade

$\square$ necessita de ajuda

$\square$ não é capaz

3) Ele (Ela) é capaz de esquentar a água para o café e apagar o fogo?

$\square$ normal

$\square$ faz com dificuldade

$\square$ necessita de ajuda

$\square$ nunca o fez, mas poderia fazê-lo agora

$\square$ nunca o fez e agora teria dificuldade $\square$ nunca o fez, mas poderia fazê-lo agora

$\square$ nunca o fez e agora teria dificuldade 
$\square$ não é capaz

4) Ele (Ela) é capaz de preparar uma comida?
$\square$ normal
$\square$ faz com dificuldade
$\square$ nunca o fez, mas poderia fazê-lo agora
$\square$ necessita de ajuda
$\square$ nunca o fez e agora teria dificuldade
$\square$ não é capaz

5) Ele (Ela) é capaz de manter-se em dia com as atualidades, com os acontecimentos da comunidade ou da vizinhança?

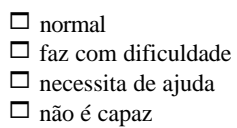

$\square$ necessita de ajuda

$\square$ nunca o fez mas poderia fazê-lo agora $\square$ nunca o fez e agora teria dificuldade

6) Ele (Ela) é capaz de prestar atenção, entender e discutir um programa de rádio ou televisão, um jornal ou uma revista?

$$
\begin{aligned}
& \square \text { normal } \\
& \square \text { faz com dificuldade } \\
& \square \text { necessita de ajuda }
\end{aligned}
$$

$\square$ não é capaz

$\square$ nunca o fez, mas poderia fazê-lo agora
$\square$ nunca o fez e agora teria dificuldade

7) Ele (Ela) é capaz de lembrar-se de compromissos, acontecimentos familiares, feriados?

$$
\begin{aligned}
& \square \text { normal } \\
& \square \text { faz com dificuldade } \\
& \square \text { necessita de ajuda } \\
& \square \text { não é capaz }
\end{aligned}
$$

$\square$ nunca o fez, mas poderia fazê-lo agora $\square$ nunca o fez e agora teria dificuldade

8) Ele (Ela) é capaz de manusear seus próprios remédios?
$\square$ normal
$\square$ faz com dificuldade
$\square$ necessita de ajuda
$\square$ nunca o fez, mas poderia fazê-lo agora
$\square$ não é capaz

9) Ele (Ela) é capaz de passear pela vizinhança e encontrar o caminho de volta para casa?

$$
\begin{aligned}
& \square \text { normal } \\
& \square \text { faz com dificuldade } \\
& \square \text { necessita de ajuda } \\
& \square \text { não é capaz }
\end{aligned}
$$

10) Ele (Ela) pode ser deixado (a) sozinho (a) de forma segura?

$$
\begin{aligned}
& \square \text { normal } \\
& \square \text { faz com dificuldade } \\
& \square \text { necessita de ajuda } \\
& \square \text { não é capaz }
\end{aligned}
$$

$\square$ nunca o fez, mas poderia fazê-lo agora $\square$ nunca o fez e agora teria dificuldade 
MINI-EXAME DO ESTADO MENTAL - MEEM (Folstein, Folstein \& McHugh, 1975)

\section{ORIENTAÇÃO}

\begin{tabular}{l}
\hline - Dia da semana \\
\hline - Dia do mês \\
\hline - Mês \\
\hline - Ano \\
\hline - Hora aproximada \\
\hline - Local específico (aposento ou setor) \\
\hline - Instituição (hospital, residência, clínica) \\
\hline - Bairro ou rua próxima \\
\hline - Cidade \\
\hline -Estado
\end{tabular}

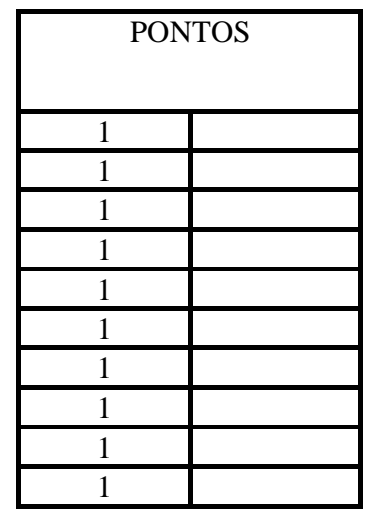

\section{MEMÓRIA IMEDIATA}

- Vaso, carro, tijolo

\section{ATENÇÃO E CÁLCULO}

$-100-7$ sucessivos

\section{EVOCAÇÃO}

- Recordar as 3 palavras

\section{LINGUAGEM}

- Nomear um relógio e uma caneta

- Repetir: "Nem aqui, nem ali, nem lá"

- Comando: "Pegue este papel com sua mão direita,

dobre ao meio e coloque no chão"

- Ler e obedecer: "Feche os olhos"

- Escrever uma frase

- Copiar um desenho

ESCORE 30

\section{SOLETRAR}

- Soletrar a palavra "mundo" de trás para a frente
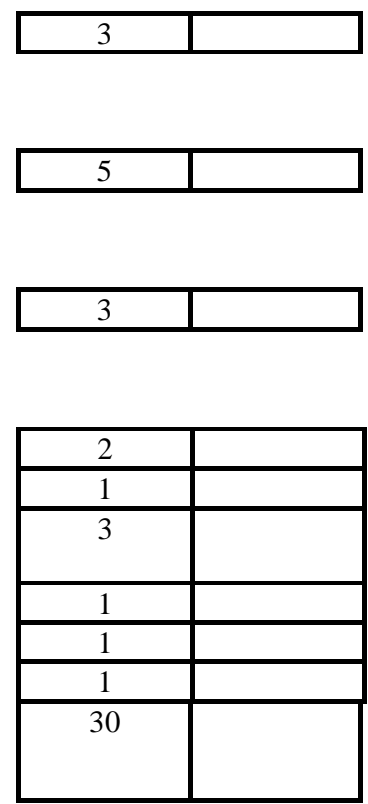


\section{FECHE OS OLHOS}

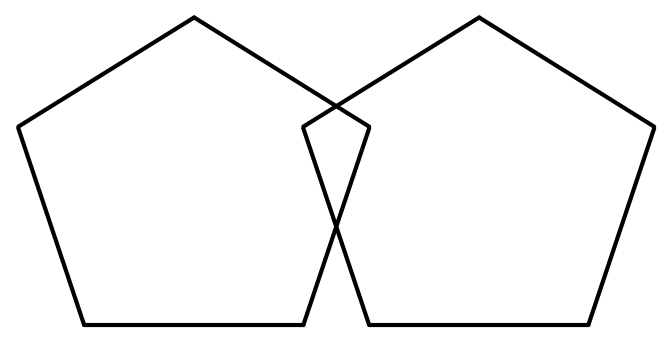

